\title{
Simulating the dynamics of individual adaptation to floods
}

\author{
Katrin Erdlenbruch*1 and Bruno Bonté ${ }^{1}$ \\ ${ }^{1}$ G-EAU, Irstea, AgroParisTech, Cirad, IRD, Montpellier SupAgro, Univ \\ Montpellier, Montpellier, France.
}

March 10

\begin{abstract}
Individual adaptation measures are an important tool for households to reduce the negative consequences of floods. Although people's motivations to adopt such measures are widely studied in the literature, the diffusion of adaptations within a given population is less well described. In this paper, we build a dynamic agent based model which simulates the adoption of individual adaptation measures and enables evaluation of the efficiency of different communication policies. We run our model using an original dataset, based on a survey in France. We test the importance of different parameters of our model by implementing a global sensitivity analysis. We then compare the ranking and performance of different communication policies under different model settings. We show that in all settings, targeted policies that deal with both risk and coping possibilities, perform best in supporting individual adaptation. Moreover, we show that different dynamic parameters are of particular importance, namely the delay between the motivation to act and the implementation of the measure and the time during which households stick to a given adaptation measure.
\end{abstract}

Keywords: flood risk, adaptation, agent based model, protection motivation theory, social network, smallworld, risk communication, flood prevention, vulnerability.

\section{Introduction}

Floods cause major damage and disruptions worldwide. In Europe, between 1980 and 2011, floods affected more than 5.5 million people and resulted in more than 2500 fatalities and over 90 billion euros in economic losses (EEA, 2012). In France, one resident out of four and one job out of three are exposed to flood risk. Between 1988 and 2013, floods were responsible for around 30 billion euros of damage in France (MEDDE, 2012a,b).

Many different policies exist to address this risk: structural measures (dykes, dams) or non-structural measures (flood retention basins) may be established with the aim of reducing hazard. National compensation schemes and private insurance policies can be used to help people to recover after a crisis and hence increase resilience. Zoning policies may be designed with the aim of reducing vulnerability (Erdlenbruch et al., 2009), e.g. by enforcing building restrictions or the adaptation of buildings in risky areas. Among the vulnerability reducing actions, some reduce the negative consequences of floods at the individual level. Households can choose, e.g. to use water-resistant materials in their homes,

\footnotetext{
*Corresponding author: Irstea, UMR G-EAU, 361 rue Jean François Breton, 34196 Montpellier cedex 5, France. Tel: +33 467046387. E-mail: katrin.erdlenbruch@irstea.fr
} 
or to store valuables upstairs. Following Blanco et al. (2017), we term such actions "individual adaptation measures" as opposed to collective mitigation measures, which reduce the probability of group loss.

Risks are reduced more effectively if adaptations at different scales are combined (Adger et al., 2005, Filatova, 2014). This may be the case when governments set up information policies or price signals for private stakeholders or when they support market based instruments (Filatova, 2014). In this paper, we focus on how individual adaptation measures can be promoted by public communication policies. To situate our approach with respect to the risk governance model (Aven and Renn, 2010, IRGC, 2005), we assume that risk appraisal and concern assessment as well as risk characterisation and tolerability assessment are carried out at the individual level. Different communication policies representing different regulatory styles are implemented by the risk management institutions, e.g. local water basin manager or the national ministry of the environment.

Some individual adaptation measures have been shown to be particularly cost efficient, reducing the ratio of total damage to total building values by nearly half (see Kreibich et al. (2005) for a study in Germany, Poussin et al. (2015) for a study in France, and Botzen et al. (2009) for a study in the Netherlands). Many other advantages of individual adaptation are discussed in the literature, among which the fact that they may help to maintain awareness about flood risk among people (Richert et al., 2017).

Although the reasons to adopt individual adaptation measures are relatively well covered in the literature, little is known about the dynamic aspects of adaptation: how long do people stick to a chosen measure? Once people have the intention to adapt, how quickly do they implement the measure? At a more aggregated level: how rapidly do adaptation measures expand within a population? Our main research question in this paper is thus: how important are the dynamic aspects of the adoption of individual adaptation measures and how do they influence the predictions about the effectiveness of public policies supporting individual adaptation? To answer this question, we built an empirically based dynamic simulation model of the adoption of individual adaptation measures.

To study the driving forces of the adoption of individual adaptation measures, many studies have used the protection motivation theory (PMT) because it focuses on two complementary elements of risk perception, threat appraisal and coping appraisal. The theory was proposed by Rogers (1975) and originally applied in the health domain (see Milne et al. (2000) for a metanalysis). Following Grothmann and Reusswig (2006), the framework was extended to explain the adoption of individual adaptation measures against floods. Authors in various countries have used this approach, e.g. in Germany (Bubeck et al., 2013, Grothmann and Reusswig, 2006), Great Britan (Glenk and Fischer, 2010), Vietnam (Reynaud et al., 2013), and France (Poussin et al., 2014, Richert et al., 2017). Similarly, this approach has been used to explain the adoption of individual adaptation in the face of drought events (van Duinen et al., 2014, van Duinen et al., 2015). This is the framework we use in the following.

Several studies have drawn attention to the fact that risk perceptions and adaption behaviour should be modelled in a dynamic setting (Bubeck et al., 2012a,b). For example, risk perception satisfactorily explains the intention to adopt individual adaptation measures but not necessarily the presence of such measures, because there could be a feedback effect, which decreases risk perceptions once the measures are adopted (Bubeck et al., 2012a, Richert et al., 2017). On the other hand, households may decide to abandon measures if no flood occurs for a while, as the experience of flood events is an important element in explaining past implementation of individual adaptation measures (Osberghaus, 2017). Despite these results, longitudinal data on adaptation behaviour are scarce and time consuming to collect (Osberghaus, 2017). One way to investigate the dynamics of adaptation despite this missing data is to use simulation models, such as agent based models. 
Agent based models (ABM) make it possible to test hypotheses concerning the relationship between individual behaviours and macroscopic regularities (Epstein and Axtell, 1996), for instance the rate of adaptation in a population as the outcome of many individual adaptation decisions. ABMs can also be used to explore non equilibrium dynamics (Epstein and Axtell, 1996) and to test the importance of sets of parameters for which empirical data are missing. It is thus an interesting tool to test changes in rates of adaptation in the population as a function of different dynamic parameters of individual adaptation. Finally, ABMs can be easily combined with spatial models and can consequently represent the networks and interactions among individuals which are crucial in social systems. In this paper, we consider a typical social network, exhibiting the small-world characteristics, which have been shown to exist in many social interactions (Watts and Strogatz, 1998). Agent based models have already been applied to flood risk management. Dawson et al. (2011) for example built a flood-incidence model which mimics the short-term reaction of individuals during a flood, applied to the coastal town of Towyn, UK. Filatova (2015), Filatova et al. (2011) and Dubbelboer et al. (2017) modelled the long-term effect of flood risk on the housing market. Other studies showed the influence of different behavioural assumptions on changes in land-use or in investment decisions: for example, Filatova et al. (2011) show how a skewed risk perception distribution leads to more high valued development in risky coastal zones. Haer et al. (2016a) investigated three economic decision models for investments in loss-reducing measures in the context of river flooding. Finally, Haer et al. (2016b) and van Duinen et al. (2016) combined protection motivation theory and agent based models of adaptation diffusion, applied to drought risks in van Duinen et al. (2016) and flood risk in Haer et al. (2016b).

The model built by Haer et al. (2016b) is closest to ours. They tested the effectiveness of four different flood-communication policies in promoting individual adaptation measures in the Dutch Rotterdam-Rijnmond area. Risk communication can be top down or people centered, i.e. tailored to the specific needs of an individual. One example of top down policies is when governments communicate about risk zoning. One example of people centered policies is when experts advise homeowners how to make their home flood-proof. The information provided in these communication campaigns can deal with the occurrence and consequences of flood risk or it can describe actions and measures that people can use to cope with the risk. One could for example imagine a photo exhibition showing past events to describe the risk and advise on how to behave in the case of a crisis to describe how to cope with different types of risk. Haer et al. (2016b) show that polices perform best if the information is people centered and if it deals with both the risk and coping with risk.

Our model differs from their model in three main ways: first, whereas Haer et al. (2016b) construct an artificial society based on data found in Bubeck et al. (2013), we use our own dataset and only model households on which we have detailed information. Second, Haer et al. (2016b) construct a social network based on the characteristics of networks in the Netherlands, we construct a spatially explicit small-world network on the basis of our data. Third, and most importantly, we adapt our model to be able to represent two important dynamic features: the average delay of implementation of the measure and the average adaptation duration. We analyse the importance of the different parameters of our model by comparing it to a similar aggregate model and by performing a global sensitivity analysis. We then investigate the impacts of the four communication policies in this model, considering different dynamic adaptation configurations. In particular, we show that the delay of implementation is the most influential parameter, next to the duration of individual adaptation measures.

The paper is organized as follows: In section 2, we describe the survey and the empirical data we use. In section 3, we present our empirical decision model: we first describe how 
protection motivation can be represented as the probability to adopt adaptation measures and we then present the results of estimating this probability from the underlying dataset. In section 4, we describe the model of adaptation diffusion, with a special emphasis on the construction of the social network and the dynamic parameters. We also compare the individual based model to an aggregate model to gain some additional insights into the dynamics modelled and to demonstrate the interest of individual-based modelling in this specific case. In section 5 , we present the experimental plan of our simulations. In section 6 , we present the results: first we assess the importance of different parameters of our model, by comparing it to the aggregate model and by implementing a sensitivity analysis. We then observe how different model configurations affect the ranking and efficiency of the four communication policies. This allows us to represent the diffusion of individual adaptation measures under different dynamic settings. Finally, in section 7 we present our conclusions. Figure 1 summarizes the main steps of our work.

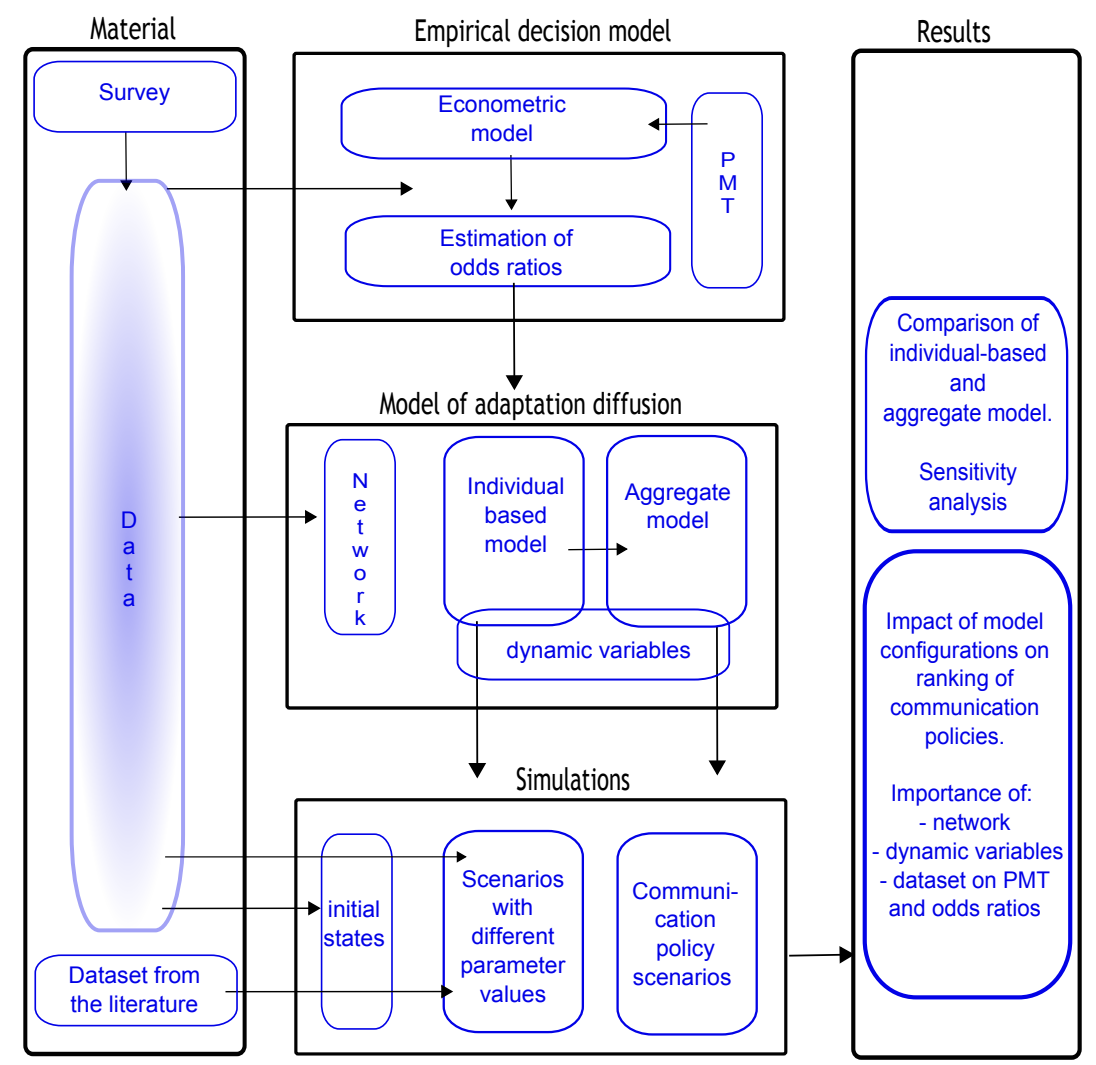

Figure 1: Graphic organizer of the underlying work.

\section{Material}

\subsection{Survey and geographical sampling}

Our data is based on a survey of 331 households in the Aude and the Var departments in the South of France, conducted in summer 2015. The distribution of the surveyed households and their location in the floodplains are shown in the maps in Figure 2. All the sampled municipalities were hit by important floods in the years preceding the survey: in the Var department in 2010, 2011, 2013 and 2014, and in the Aude department in 1999 and 2014. Some municipalities are flooded regularly while others were hit only by major floods, namely in the Aude departement in 1999 and in the Var department in 2010. The 
majority of the respondents live in flood-prone areas: $80 \%$ of the respondents had already experienced a flood, as defined in the survey by "the flood reached your street". About half of the respondents live in big municipalities, the other half in rural municipalities. In the survey, we also collected information on location of the households in the flood prone areas, the characteristics of their homes, their risk perceptions and their behaviour during a flood and in preparation of future floods. For more details on the survey, see Richert et al. (2017).

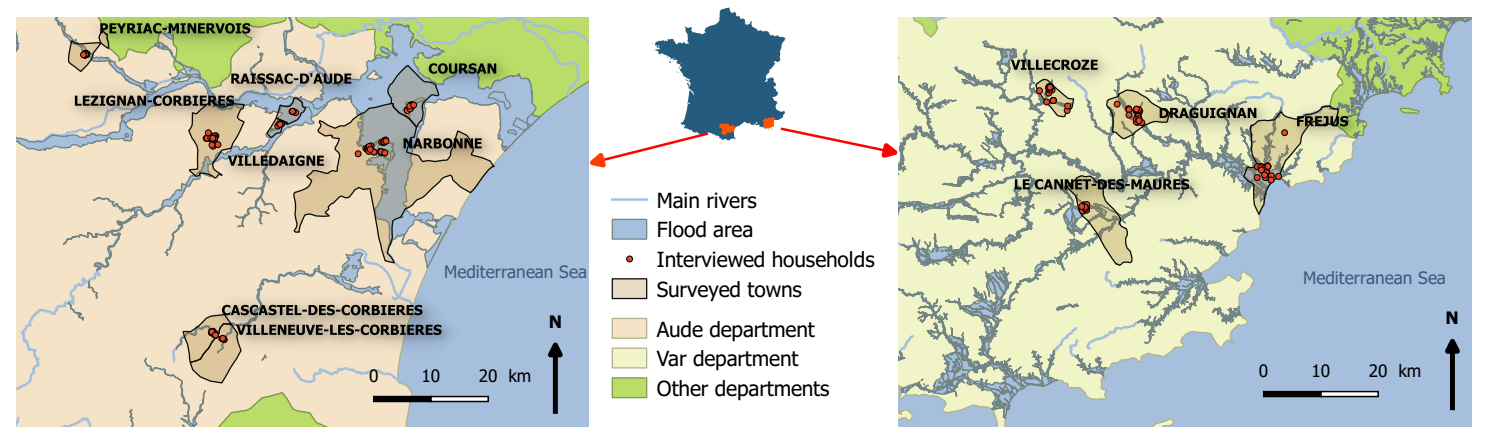

Figure 2: Map of sampled municipalities and surveyed households in relation to flood prone areas in the South of France, Aude department on the left, Var department on the right.

\subsection{Data}

\subsubsection{Adaptation measures}

Eleven main adaptation measures were identified in the survey, see Table 1. For the purpose of this study, we distinguish permanent from non-permanent measures. Permanent measures are features of the structure of homes, such as raised ground floors or raised crawl spaces, also termed structural measures. Non-permanent measures may either be temporary or reversible.

\begin{tabular}{lll}
\hline \multicolumn{1}{c}{ Permanent measures } & \multicolumn{1}{c}{ Reversible measures } & Temporary measures \\
\hline $\begin{array}{l}\text { Raised ground floor, raised } \\
\text { crawl space }\end{array}$ & $\begin{array}{l}\text { Use of water resistant } \\
\text { materials (for the floor } \\
\text { and/or the walls) }\end{array}$ & Slot-in flood barriers \\
$\begin{array}{l}\text { Opening on the roof to } \\
\text { facilitate evacuation }\end{array}$ & $\begin{array}{l}\text { All main rooms (kitchen, } \\
\text { bedrooms, living-room) } \\
\text { located upstairs }\end{array}$ & Pumps \\
$\begin{array}{l}\text { Watertight doors and } \\
\text { windows }\end{array}$ & $\begin{array}{l}\text { Electrical wiring and systems } \\
\text { and/or boiler installed higher } \\
\text { up on the walls }\end{array}$ & Valuables stored upstairs \\
$\begin{array}{l}\text { Measures to improve water } \\
\text { flow }\end{array}$ & Sewer non-return valves & \\
\hline
\end{tabular}

Table 1: Permanent and non-permanent (i.e. reversible and temporary) measures revealed during the survey. 
Temporary measures are those that depend on the behaviour of the household: using slotin flood barriers or pumps, or storing valuables upstairs. Reversible measures imply some installation but have to be maintained or may be easily removable, such as having all main rooms upstairs, or installing electrical wiring and boilers further up on the walls. As revealed by some additional qualitative surveys we led, temporary measures can disappear after a few years, whereas reversible measures may last until the renewal of most of the installations, about 10 years.

\subsubsection{Household attributes}

Sociodemographic household attributes Some general sociodemographic characteristics of the household sample are summarized in Table 2. The final sample used is based on 272 individuals and is representative of the municipal population in terms of age and gender. It is also balanced in terms of geographical distribution and size of municipalities. Finally, it is sufficiently diversified in terms of education level and homeownership. For more details see Richert et al. (2017).

\begin{tabular}{ccc}
\hline Variable & Category & $\begin{array}{c}\text { Sample } \\
\text { distribution }\end{array}$ \\
\hline \multirow{2}{*}{ Gender } & Male & $46.7 \%$ \\
& Female & $53.3 \%$ \\
\hline \multirow{2}{*}{ Age } & $<30$ & $17.6 \%$ \\
& $30-44$ & $21.3 \%$ \\
& $45-59$ & $25.0 \%$ \\
Education level & $60-74$ & $26.5 \%$ \\
& Less than a high school diploma & $9.6 \%$ \\
\hline \multirow{2}{*}{ Ownership of the home } & High school diploma or higher diploma & $51.1 \%$ \\
& Home owners & $48.9 \%$ \\
\hline \multirow{2}{*}{ Size of the municipality } & Others & $63.2 \%$ \\
of residence & Resident of a municipality with less than & $36.8 \%$ \\
\hline \multirow{2}{*}{ Department } & 10,000 inhabitants & $52.6 \%$ \\
& Resident of a municipality with more than & $47.4 \%$ \\
\hline
\end{tabular}

$\mathrm{N}=272$

Table 2: Distribution of sociodemographic variables in the sample.

Household attributes related to the protection motivation theory The attribute variables used in our model are listed in Table 3, see also Richert et al. (2017) for a more detailed description. All attitude variables were rescaled between 1 and 5. The variable, "Well-being in municipality" which is measured as "Do you feel well in your municipality? ("Not well at all-1", "Not really well-2", "Neither well nor not well-3", "Well-4", "Very well$\left.5^{\prime \prime}\right)$ is included as a proxy for "perceived benefits of living in a flood prone area". Indeed, in the protection motivation theory, perceived benefits can temper the threat appraisal. The variable "perceived costs" is measured through the proxy: "For each measure listed, 
are its use and its maintenance constraining?" ("Not at all-1" "Not really constraining-2" "Don't know-3" "Yes a little constraining-4" "Yes, very constraining").

\begin{tabular}{|c|c|c|c|}
\hline Variable & $\begin{array}{c}\text { Mean } \\
\text { (Std dev.) }\end{array}$ & Question & Scale \\
\hline $\begin{array}{l}\text { Perceived } \\
\text { probability }\end{array}$ & $\begin{array}{c}3.39 \\
(1.12)\end{array}$ & $\begin{array}{l}\text { "How do you assess the following scenario: } \\
\text { 'your municipality will be flooded at least } \\
\text { once in the next } 10 \text { years'?" }\end{array}$ & $\begin{array}{l}\text { From } 1 \text { ("impossible") } \\
\text { to } 5 \text { ("certain") }\end{array}$ \\
\hline $\begin{array}{c}\text { Perceived } \\
\text { consequences }\end{array}$ & $\begin{array}{c}3.47 \\
(1.40)\end{array}$ & $\begin{array}{c}\text { "In the case of flooding, how do you assess } \\
\text { the following scenario: 'the water will reach } \\
\text { your street'? " }\end{array}$ & $\begin{array}{c}\text { From } 1 \text { ("impossible") } \\
\text { to } 5 \text { ("certain") }\end{array}$ \\
\hline $\begin{array}{c}\text { Perceived } \\
\text { self efficacy }\end{array}$ & $\begin{array}{c}2.50 \\
(1.10)\end{array}$ & $\begin{array}{c}\text { "To what extent do you agree with the } \\
\text { following statement: 'I do not believe that I } \\
\text { am able to avoid the consequences of floods } \\
\text { in my household. I have no control over such } \\
\text { events.'?" }\end{array}$ & $\begin{array}{c}\text { From } 1 \\
\text { ("strongly agree") } \\
\text { to } 5 \\
\text { ("strongly disagree") }\end{array}$ \\
\hline $\begin{array}{l}\text { Perceived } \\
\text { efficacy of } \\
\text { measure }\end{array}$ & $\begin{array}{c}3.57 \\
(0.86)\end{array}$ & $\begin{array}{l}\text { "For each measure listed below, how } \\
\text { effective do you think it will be in preventing } \\
\text { the negative consequences of floods?" }\end{array}$ & $\begin{array}{c}\text { From } 1 \\
\text { ("not at all effective") } \\
\text { to } 5 \\
\text { ("very effective") }\end{array}$ \\
\hline $\begin{array}{l}\text { Past flood } \\
\text { experience } \\
\text { appraisal }\end{array}$ & $\begin{array}{c}2.59 \\
(1.40)\end{array}$ & $\begin{array}{c}\text { "How do you assess the seriousness of the } \\
\text { consequences of the reference flood for your } \\
\text { household?" }\end{array}$ & $\begin{array}{l}\text { From } 1 \text { ("not or for } \\
\text { people who have not } \\
\text { experienced a flood) } \\
\text { to } 5 \text { ("extremely } \\
\text { serious") }\end{array}$ \\
\hline $\begin{array}{c}\text { Perceived } \\
\text { benefits }\end{array}$ & $\begin{array}{c}4.30 \\
(0.79)\end{array}$ & "How well do you feel in your municipality?" & $\begin{array}{c}\text { From } 1 \text { ("not well at } \\
\text { all") to } 5 \text { ("very } \\
\text { well") }\end{array}$ \\
\hline $\begin{array}{l}\text { Perceived } \\
\text { costs }\end{array}$ & $\begin{array}{c}2.94 \\
(1.35)\end{array}$ & $\begin{array}{l}\text { "For each measure listed its use and its } \\
\text { maintenance are they constraining?" }\end{array}$ & $\begin{array}{l}\text { From } 1 \text { ("not at all") } \\
\text { to } 5 \text { ("extremely } \\
\text { serious") }\end{array}$ \\
\hline
\end{tabular}

$\mathrm{N}=272$

Table 3: Summary of PMT data: variable name corresponding to household attribute, mean value and standard deviation in the sample, question used in the questionnaire, possible values for each attribute.

Social network and adaptation status For the social network variable, we created a variable that counts the number of adapted neighbours. We distributed the interviewed individuals according to their geographic location and created a directed network of the 15 nearest neighbours (see section 4). Considering a group of 1 to 20 people when deciding on flood adpation measures seemed a good assumption to us (see also Haer et al. (2016b)). The Social Network variable varies from 1 ("no adapted neighbour") to 5 ("only adapted neighbours") with a mean of 2.54 and standard deviation of 0.82 .

To define the initial adaptation status of our network, we used the following information: In our sample of 272 individuals, 183 individuals have at least one non-permanent adaptation measure in their home. Hence $67 \%$ of the households are adapted in some way. However, 
some of these adaptations may have already been present when the family moved in. Indeed, only 66 individuals, or $24 \%$, state that they implemented the measure themselves. In the following, we consider that the initial adaptation status is $67 \%$. We anticipate that adaptation trajectories will decrease in the beginning, as some of the households had not chosen to implement the adaptation measures and may not be motivated to do so.

\section{The empirical decision model}

\subsection{Explaining protection motivation in an econometric model}

The general setup of the model The empirical decision model is based on the protection motivation theory by Rogers (1975). Households' or individuals' adaptation behaviour ${ }^{1}$ is explained by several variables, as shown in Figure 3 (blue rectangles from top to bottom): First, individuals may evaluate the experience of past flood events differently. Secondly, they perceive the threat of flooding differently: the probability and consequences of future events as well as potential benefits of living in risky areas. Thirdly, they appraise their coping capacity differently: the efficacy of adaptation measures and their own efficacy when adapting, as well as the perceived costs of implementation. Finally, they are part of social networks in which varying numbers of other members have adapted, which may influence their own behaviour. The impact of each variable on protection motivation can be positive (represented by green arrows, with a plus sign) which means the higher the value of the variable, the greater the response variable, or negative (represented by red arrows, with a negative sign), which means the higher the value of the variable, the lower the response variable. For example: the greater the perception of the probability of occurrence of a flood, the greater the motivation to act. Next to the protection motivation, these variables can also explain non-protective responses, such as wishful thinking and denial. For example, a high coping appraisal will reduce non-protective responses which in turn increases protection motivation. Here we represent the most common links described in the literature (Richert et al., 2017). To keep the graph intelligible, we do not include feedback effects, which may exist: e.g. the adoption of adaptation measures may in turn lead to lower threat appraisal, see (Richert et al., 2017). Each of these variables, or attributes, is measured through attribute levels (see Table 3), which can be interpreted as individual attitudes. Protection motivation may be transformed into the implementation of adaptation measures. However, actual barriers, such as high implementation costs or administrative problems, can impede the action.

\footnotetext{
${ }^{1}$ Here and in the following, we use either "individual" or "household" to refer to the data we collected on individuals concerning their household.
} 


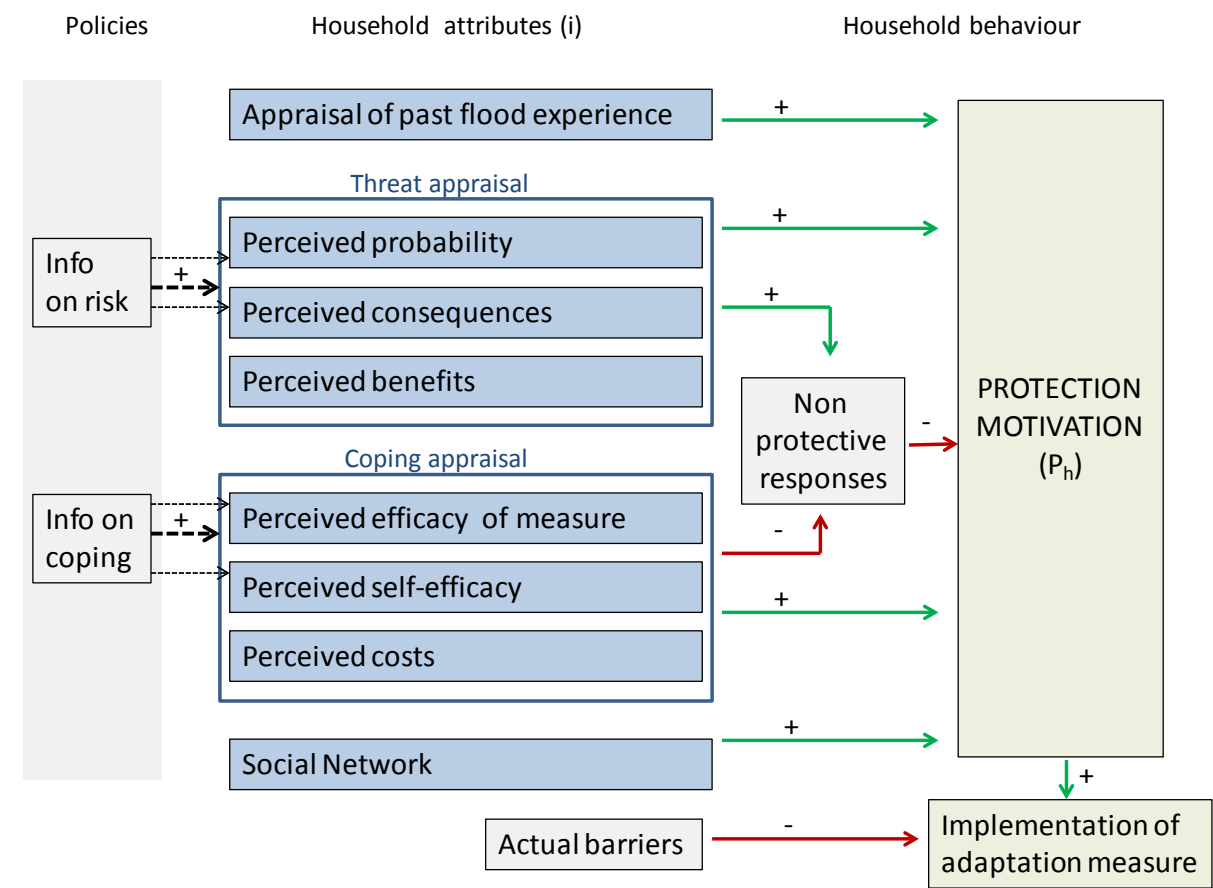

Figure 3: General representation of the impact of households' attributes on households' behaviour according to the Protection Motivation Theory, assuming the presence of communication policies.

The econometric model The motivation to take protective responses can be measured econometrically by estimating the probability to adopt adaptation measures as a function of the explanatory variables. The probability to adopt adaptation measures for each household, $P_{h}$ can be written as a function of the household's attribute levels and the odds ratios for implementation:

$$
P_{h}=\frac{C \Pi_{i=1}^{I} o r_{i}^{a_{i, h}}}{C \Pi_{i=1}^{I} o r_{i}^{a_{i, h}}+1},
$$

where $i$ is the attributes, $I$ is the full set of attributes, $o r_{i}$ is the odds ratio for each attribute as computed in a logistic regression, $C$ is the constant of the logistic regression and $a_{i, h}$ are each attribute's level. Attribute levels are household specific and can be interpreted as household's attitudes. The odds for some event expresses the likelihood that the event will take place divided by the likelihood that it will not. In the logistic regression, the odds ratio indicates the change in odds of the explanatory variable for a unit change in the dependent variable. ${ }^{2}$

Communication policies We also consider the possibility of communication policies (see left-hand side of Figure 3). Following Haer et al. (2016b), communication strategies on flood risk can take four forms: top down policies on risk ("td-r"), top down policies on risk and coping ("td-rc"), people centered policies on risk ("pc-r") and people centered policies on risk and coping ("pc-rc"). Top down policies on risk ("td-r") increase the attribute level of both perceived probability and perceived consequences by two units each, for all individuals who are reached by the policy. Top down policies on risk and coping (td-rc) increase the attribute level of four variables: perceived probability and perceived

\footnotetext{
${ }^{2}$ If more than two levels are possible for one variable, the odds are computed for passing from one level to the next.
} 
consequences as well as the perceived efficacy of the measure and perceived self-efficacy, by one unit each. Like in Haer et al. (2016b), we assume that the magnitude of change in attitude is the same for the two communication strategies. Only the way in which attitude is changed differs. This makes the policies comparable. People centered communication policies function in a similar way, increasing the attribute levels of either risk or risk and coping variables by at most 4 points. However, they first target the variables which have the lowest attribute levels and hence the most need to be increased. If an individual already has the maximum possible attribute level, the policy has no additional impact. All households have a combined probability $p$ to be targeted by a communication policy and to take into the information obtained into consideration. Following Haer et al. (2016b), we chose $p=0.16$.

\subsection{Estimation of odds ratios describing the probability to adopt}

We run a logistic regression of an individual's intention to implement non-permanent adaptation measures on the household's attributes listed in Table 3. Table 4 summarizes the regression results and lists the corresponding odds ratios, with $95 \%$ confidence intervals. ${ }^{3}$

Table 4: Logistic regression explaining planned non-permanent measures with variables from the Protection Motivation Theory and the social network variable.

\section{Logit estimation}

Variable
Odds ratio

$95 \%$ confidence intervals

\begin{tabular}{lccccc} 
& Coefficient & Std Err. & Lower & Odds ratio & Upper \\
\hline Perceived probability & 0.07 & $(0.17)$ & $(0.78)$ & 1.08 & $(1.49)$ \\
Perceived consequences & $0.61^{* * *}$ & $(0.17)$ & $(1.32)$ & 1.83 & $(2.54)$ \\
Perceived self-efficacy & 0.22 & $(0.16)$ & $(0.92)$ & 1.24 & $(1.69)$ \\
Perceived efficacy of measure & 0.03 & $(0.21)$ & $(0.68)$ & 1.03 & $(1.56)$ \\
Appraisal of past flood experience & $0.41^{* * *}$ & $(0.13)$ & $(1.16)$ & 1.51 & $(1.96)$ \\
Perceived benefits & $0.53^{* *}$ & $(0.24)$ & $(1.07)$ & 1.70 & $(2.71)$ \\
Perceived costs & 0.06 & $(0.13)$ & $(0.82)$ & 1.06 & $(1.38)$ \\
Social Network & $0.43^{* *}$ & $(0.21)$ & $(1.01)$ & 1.54 & $(2.34)$ \\
Intercept & $-9.29^{* * *}$ & $(1.84)$ & $(0.00)$ & 0.00 & $(0.00)$ \\
\hline
\end{tabular}

Nagelkerke $\mathbf{R}^{2}$

0.34

$\mathrm{N}=272$. Significance levels: ${ }^{*} \mathrm{p}<0.1 ;{ }^{* *} \mathrm{p}<0.05 ;{ }^{* * *} \mathrm{p}<0.01$.

Four of the variables have significant positive impacts: when perceived consequences, the appraisal of past flood experience, the wellbeing or the adaptation level in the social net-

\footnotetext{
${ }^{3}$ Note that this regression takes into account the possible existence of feedback effects between risk perception and adaptation because the dependent variable relates to planned measures.
} 
work increase, the probability to adopt adaptation measures increases. The odds ratio indicates the change in the odds of the explanatory variable for a unit change in the dependent variable. For instance, for one unit change in perceived consequences the probability of adoption will be multiplied by 1.83 .

\section{A Model of adaptation diffusion}

In this section, we describe the model of diffusion of individual adaptation measures. We first present the individual based model, its functioning and main variables. We also compare the model with a corresponding aggregate model which provides some additional insights into the dynamic behaviour of the model.

\subsection{An individual based model}

\subsubsection{Agents}

Agents are geographically situated and connected through a social network. Agents are heterogenous in their attitudes, i.e. the attribute levels of the PMT variables listed in Table 3. Every year, agents decide whether or not to adopt an individual adaptation measure.

\subsubsection{Construction of the small world network}

In the following, we build a static social network that takes into account the spatial distribution of our data. The network variable plays a particular role in our individual based model. In theory, the higher the proportion of neighbours in the household's network who have adapted, the higher its attribute level for the social network variable. Clearly, this variable will depend greatly on two factors: the type of network under consideration and the number of neighbours considered. We test the impact of these two factors in the following section. As a reference case, we use a network with the "small-world" properties of human social networks. In their seminal paper on small-world networks, Watts and Strogatz (1998) describe the small-worldness of networks as a combination of both a high clustering coefficient, $C$, and a low path length, $L$, (relative to a regular network with the same mean degree). Let us describe the steps of the construction of this social network. We first build a network from the spatial distribution of our data by linking each node with its $n$ nearest neighbours and randomly adding links between isolated clusters in order to get a closed network. As described in the data section, we chose $n=15$. From this network, which has a high clustering coefficient and a high mean path length, we adopt the rewiring method used by Watts and Strogatz (1998) to obtain the characteristics of a small world. Namely, each link has a probability of $p_{r}$ to be removed and replaced by another randomly chosen link. The rewired network has a lower mean path length and a higher clustering coefficient than the original network. In this procedure, $C$, decreases less rapidly than $L$.

We repeat this rewiring process for various probability values, $p_{r}$ from 0.001 to 1 , in order to choose a rewiring probability, and an associated rewired network, for which the clustering coefficient $(C)$ is still high and the average path length $(L)$ is already low. Figure 4 presents all the probabilities tested and associated values obtained for $C$ and $L$ relative to regular networks with the same degree. Finally, we chose the network obtained for $p_{r}=0.015$ identified by the red vertical line in Figure 4 . Figure 5 shows the network obtained by linking each households to the 15 geographically closest households surveyed (on the left) and the network obtained after rewiring and used for the simulations (on the right). 


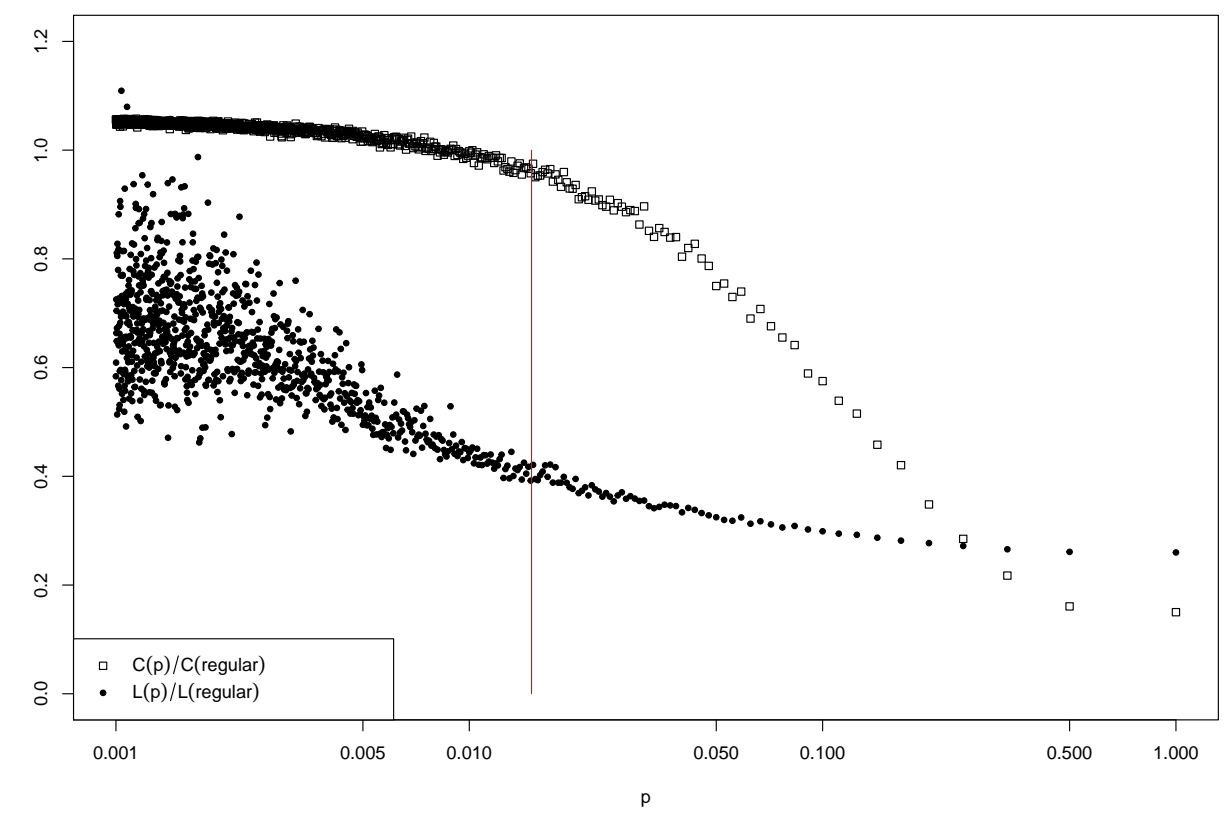

Figure 4: Clustering coefficient, C, and average path length, L, of the network as a function of rewiring probability according to the procedure proposed by Watts and Strogatz 1998.
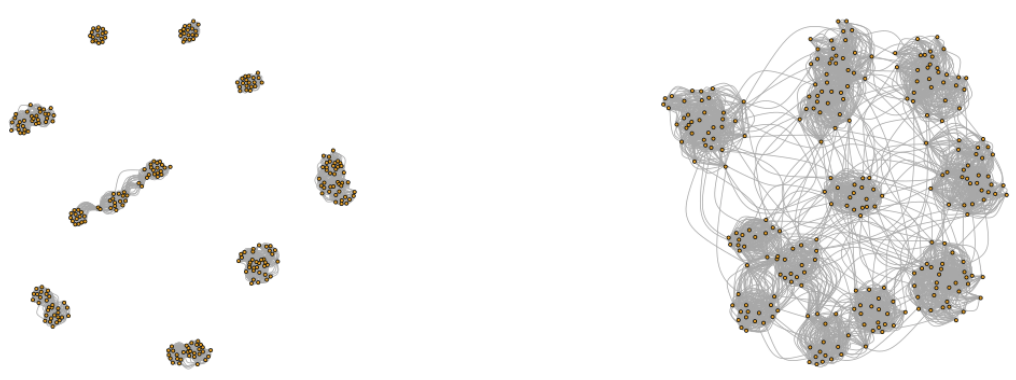

Figure 5: Network of closest households computed from household locations (on the left) and network of households' acquaintances obtained after rewiring and used in the simulations (on the right). The positions of the points in the graphs are optimized for better visualization of the networks (minimum crossing between edges, etc.) and not related to the geographical positions of the households. 


\subsubsection{Introduction of time and processes in the model}

In order to better account for the dynamic nature of the adaptation process, we integrate the following aspects in the model.

The time from motivation to action First, it should be recalled that $P_{h}$ is the probability that an individual is motivated to adopt an adaptation measure. Because we do not know when motivated individuals will transform this motivation into action, we consider that a motivated individual will really be adapted after an average of $N$ consecutive years during which he or she is motivated to adapt. Thus, the probability that a motivated individual will adapt each year is: $P_{s}=\frac{1}{N}$.

The duration of adaptation Adaptation measures may be non-permanent. Households may forget to implement the measures, e.g. no longer storing valuables upstairs, or the measure itself may fail, e.g. sewer non-return valves may age. In this paper, we only model non-permanent adaptation. We consider that on average, adaptation duration is $b$ years. Hence, on average, the probability that an adapted individual will abandon adaptation is $1 / b$.

Adoption and abandoning of measures Adaptation takes place if a randomly drawn value between 0 and 1 is smaller than the household's probability of adopting adaptation measures, $P_{h}$. Some households that were previously adapted may decide to abandon their adaptation measures. If they do so, they reset the attribute level of the perceived probability variable to zero.

The general scheduling of the model The general scheduling of the model is as follows: every year, households decide in a random order whether or not to adopt an individual adaptation measure. Next, some households may be reached by a yearly communication policy which increases several of their attitude variables, depending on the policy. Finally, all households update their attribute levels because they observe their social network and update their attitude as a function of the number of adapted neighbours in the network.

\subsection{The corresponding aggregate model}

A similar aggregate model of adaptation diffusion can be written as follows. Call $x(t)$ the percentage of adapted households and let $t$ be time. We have:

$$
x(t+1)-x(t)=\frac{1}{N} P_{h}(1-x(t))-\frac{1}{b} x(t),
$$

where $N$ is the average delay of implementation of the measure, i.e. the time to transform motivation into action, and $b$ the average adaptation duration, i.e. the time households stick to a given measure. Again, $P_{h}$ is derived from the logistic regression and the probability of adoption is computed as in equation (1), except that attribute levels are no longer household specific, $a_{i, h}$ but for example taken at the mean: $a_{i}=a_{i, \bar{h}}$, hence:

$$
P_{h}=\frac{C \Pi_{i=1}^{I} o r_{i}^{a_{i}}}{C \Pi_{i=1}^{I} o r_{i}^{a_{i}}+1} .
$$

The aggregate model represents the average dynamics of the individual based model when all agents are homogenous and in absence of any spatial correlations. 
When the social network is present, we have to account for the fact that the social network variable depends on the percentage of adapted households, which is the state variable. We have:

$$
P_{h}(x(t))=\frac{C \Pi_{i=1}^{I-1} \text { or }_{i}^{a_{i}} o r_{s}^{(1+4 x(t))}}{C \Pi_{i=1}^{I-1} \text { or }_{i}^{a_{i}} \text { or }{ }_{s}^{(1+4 x(t))}+1}
$$

with $o r_{s}$, the odds ratio of the social network variable.

\section{$5 \quad$ Experimental plan and simulations}

We run numerical applications on the basis of the 272 households of our dataset, which we have distributed spatially with their $\mathrm{x}-\mathrm{y}$ coordinates and linked in a social network, as described in section 4 .

\subsection{The baseline case}

In the baseline case, the initial proportion of adapted households is 0.67 , as argued at the end of section 2. The odds ratios, $o r_{i}$, are the ones given in Table 4 in section 2 . The mean and standard deviations of households' attribute levels, $a_{i, h}$, are given in Table 3 in section 2. The distribution of their initial values is represented in the following, in Figure 6 . The social network is the small-world network described in the previous section. Moreover, in the baseline case $b=7$ and $N=1$, that is to say: adaptation lasts on average seven years and once households are motivated to take adaptation measures, they act within a year. Finally, the time horizon is 30 years.

\subsection{Experimental plan}

Our experimental plan contains the following ranges for parameter values. In the simulations, all combinations of values are considered.

1. Adaptation duration, $b$, takes values between 3 and 13 years, by steps of 1 .

2. The delay of implementation of the measure, $N$, takes values between 1 and 10 year, by steps of 1 .

3. Communication policies: we tested the impact of four communication policies, top down on risk ("td-r"), top down on risk and coping ("td-rc"), people centered on risk ("pc-r") and people centered on risk and coping ("pc-rc"), as well as the case of no communication (no-com). As described in section 3, each communication policy increases the attribute levels on risk or risk and coping by at most 4 points, with a maximum of 5 .

4. Social network: we consider the small-world network described in section 4 above, and the case without a social network. To test the impact of the social network variable, we also perform additional simulations with a network of closest neighbors and a randomly generated network. Next to the network degree $n=15$, we also test the degrees $n=5$ and $n=10$.

5. PMT data sets and initial conditions: we consider two different sets of data with their associated initial conditions. First, we use our dataset, which is based on an empirical survey in France. The initial conditions in our model are derived from our dataset. Second, we test the data described in Bubeck et al. (2013), focusing on the measures called "adaptive building use". The corresponding odds ratios can be found in Bubeck et al. (2013) and Haer et al. (2016b). Initial conditions for Bubeck et al.'s data are in part randomized, following the application by Haer et al. (2016b). 


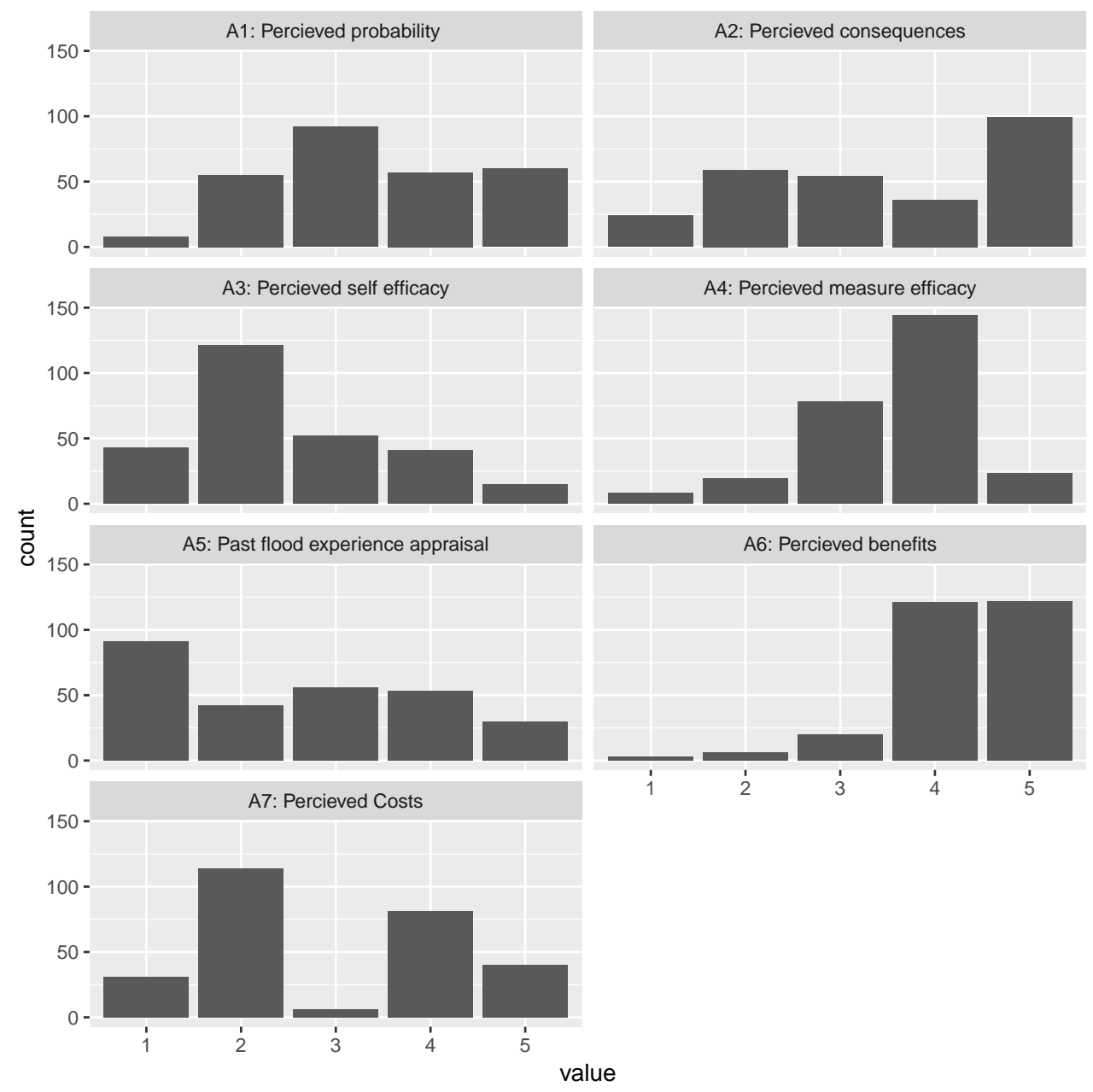

Figure 6: Initial distribution of households' attribute levels

The time horizon is of 30 years. We produced 100 iterations of each simulation. We computed the percentage of adapted households over the total number of households considered.

The simulations were run with the free opensource agent based modelling and simulation platform NetLogo (NetLogo 6.0) developed by Wilensky (1999).

\section{Results}

\subsection{Comparison between aggregate and individual based model and sen- sitivity analysis}

\subsubsection{Comparison between aggregate and individual based model}

Figure 7 shows the trajectory of adaptation levels for the case with no communication policy and no social network. The black line represents the results of the aggregate model, a mean field deterministic model, computed with average input data. The red line represents the mean value of 100 iterations of the individual based model. The red shaded area corresponds to the $20 \%$ to $80 \%$ confidence interval of outcomes and the orange shaded area to the upper and lower $20 \%$ of the simulation outcomes. We can see that computed trajectories between the aggregate and the individual based model are very close: the 
trajectory of the aggregate model remains within the $20 \%$ to $80 \%$ confidence interval of the stochastic individual based model. These results show that apart from the fact that the individual based model accounts for the heterogeneity of the population, the models are similar. One striking feature of the result is that adaptation levels are decreasing. This is due to the fact that only non-permanent adaptation measures are considered and the adaptation duration is relatively short.

Figure 8 compares the same trajectories for the baseline case, in which we have included the social network effect in both models as described in Section 4. We can see that in this case, the computed trajectories between the aggregate and the individual based model are very different: in the middle of the simulation time, the trajectory of the aggregate model exceeds the highest stochastic realisation simulated with the individual model and remains above it for the rest of the simulation. The overestimation of the speed of diffusion by aggregate models compared to the spatially explicit individual based models is well known in the study of diffusion processes and especially in epidemiology (Bonté et al., 2012). This may be due to spatial-autocorrelation of individuals able to transmit the attribute to diffuse: in a spatially explicit environment, when "infectious" individuals are close one to each other, adapted individuals have less chance of meeting non-adapted individuals than in an environment where space is not represented and individuals meet randomly. As shown in Bonté et al. (2012) the influence of spatial auto-correlation in diffusion processes depends on parameter settings.

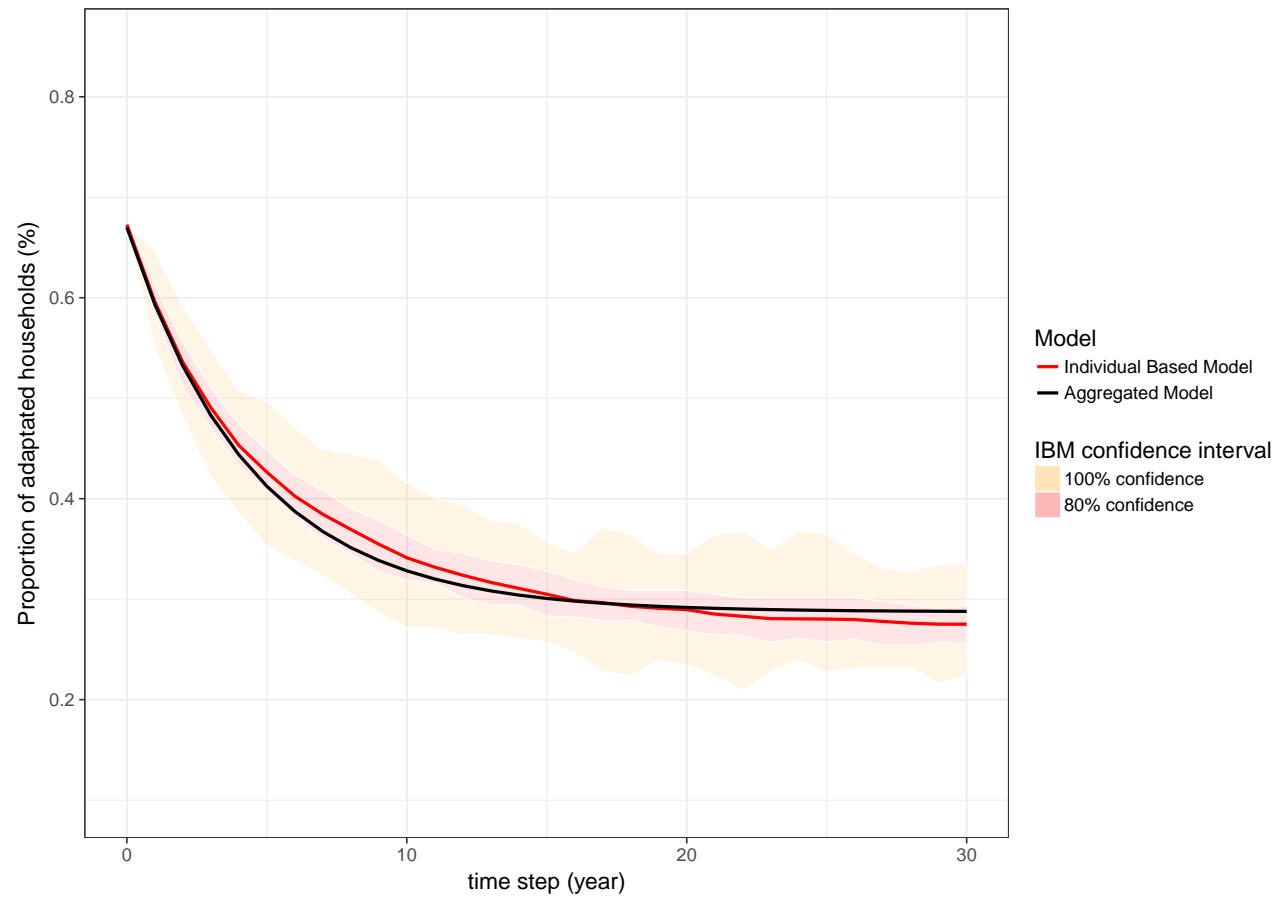

Figure 7: Adaptation trajectories for the aggregate model (black line) and the individual based model at the mean (red line), with no social network, all other parameter values as in baseline case

Despite the difference between spatially explicit and non-explicit modelling, the aggregate model can help to gain some insights into the dynamics of our model. For example, in the baseline case, we can explore how adaptation levels change when the parameter value $b$, the adaptation duration, changes, as shown on the left in Figure 9. With high values of $b$, the adaptation trajectories increase. Moreover, the greater $b$, the higher the proportion 


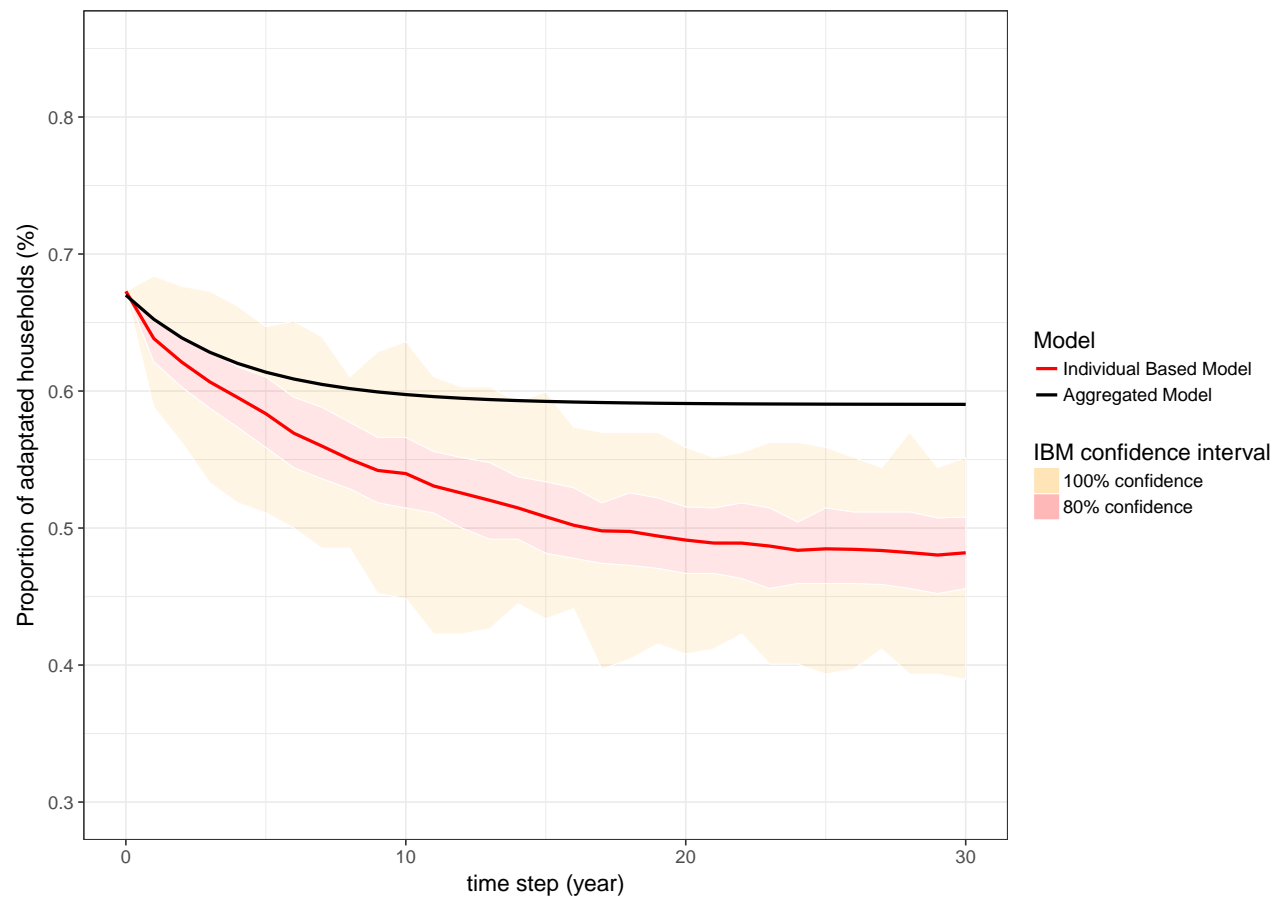

Figure 8: Adaptation trajectories for aggregate model (black line) and individual based model at the mean (red line), baseline case.

of adapted households. For example when $b=30$, the proportion of adapted households approaches $90 \%$ whereas when $b=5$ the proportion of adapted households is roughly $50 \%$. Note that the values of $b=20$ and $b=30$ may not correspond to the assumption of temporary adaptation measures, which we study in this paper. Next, we explore how adaptation trajectories change when the parameter value $N$, the delay of implementation of the measures changes, as shown on the right in Figure 9. Whereas $N=1$ leads to longterm adaptation levels of around $60 \%$, increasing $N$ leads to much lower steady states, e.g. below $30 \%$ for $N=3$ and below $10 \%$ for $N=10$. Note again that a delay of 10 years in implementation seems particularly long in our application.
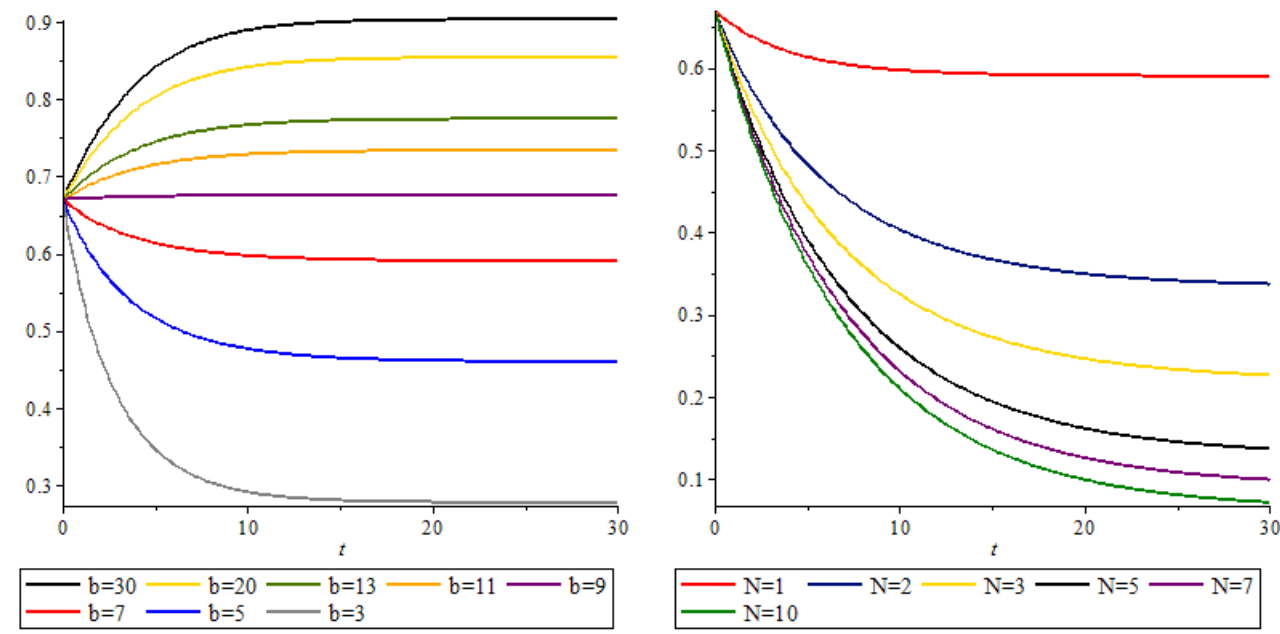

Figure 9: Adaptation trajectories for the aggregate model with different durations of adaptation, $b$, (left panel) and delay before taking action, N, (right panel). 


\subsubsection{Sensitivity analysis of the individual based model}

In order to gain a better understanding of the relative impact of the different parameters of our distributed model, we ran a global sensitivity analysis of the individual based model. Figure 10 shows the values of the first order Sobol indices computed for the modalities and parameters of the global experimental plan presented in section 5. The outcome variable is the proportion of adapted households in the population after 15 years. We can see that the parameters related to the dynamics, $N$ and $b$, have a greater impact on the result than the other parameters, the network configuration, the communication policies or the data of the PMT variables. The stochastic effect occurs because some parameter values are chosen randomly in the simulations. One explanation for the strong impact of these variables is that we consider rather high uncertainties for the dynamic parameters, varying by a factor of $10 .^{4}$

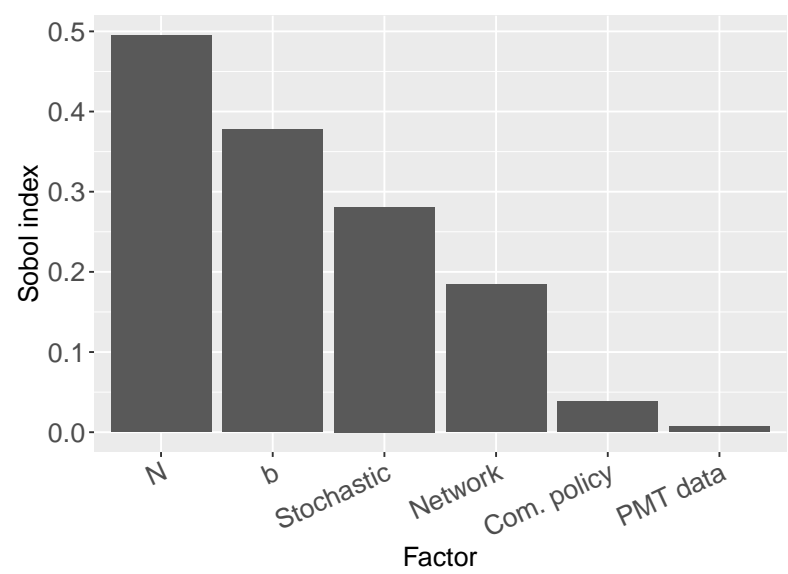

Figure 10: Sobol indices for global experimental plan. Outcome variable: proportion of adapted households after 15 years. Most influential input variables: $N$ delay of action, $b$ adaptation duration.

Among the variables tested, the average delay of implementation, $N$ is by far the most influential in explaining the variance of outcomes. Hence, if one aims at improving the dataset, most effort should be invested in evaluating the amount of time people take to really implement adaptation measures.

Figures 11 shows how the proportion of adapted households at time step 15 is distributed for different values of $N$ and $b$, based on 100 simulations. In particular, as shown in the left hand panel in Figure 11, for $N=1$, which is the value in the baseline case, the distribution of adaptation levels is right skewed and many adaptation levels cluster above $60 \%$. For $N=5$, the distribution of adaptation levels is more symmetric and mainly below $60 \%$; for $N=10$, adaptation levels are mainly below $40 \%$. The right hand panel in Figure 11 shows how adaptation levels are distributed for different values of $b$. The case of $b=7$, which is used for the baseline case, lies in the intermediate range. In this case, most adaptation levels are below $40 \%$.

\footnotetext{
${ }^{4}$ Note that the Sobol indices do not add up to one, due to the relatively small number of modalities used for the computation. However, we did a convergence study, which showed that the indices presented in Figure 10 are good approximations.
} 

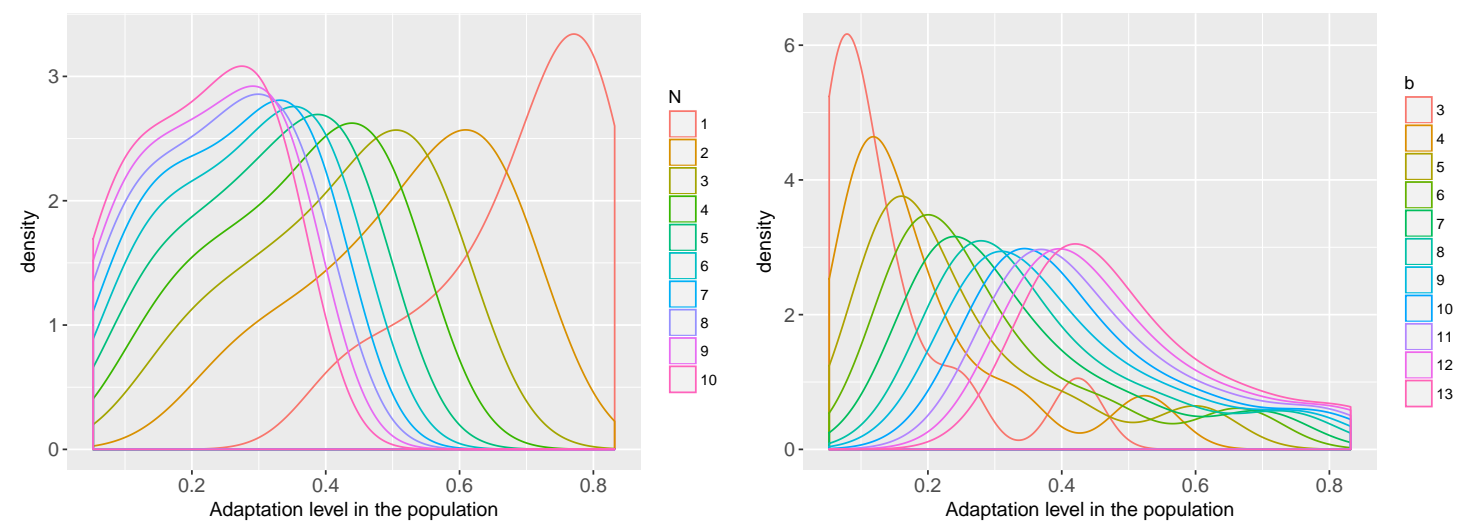

Figure 11: Distribution of the proportion of adapted households at time step 15 considering 100 simulations. Distribution represented as a function of delay of action, $N$, (left) and of adaptation duration, $b$, (right).

\subsection{The impact of model configuration on the ranking of communication policies}

Let us now study the impact of different model configurations on the ranking of communication policies.

\subsubsection{The impact of communication policies in the baseline case}

Figure 12 shows the changes in the adaptation levels over 30 years in the baseline case: the red line represents the no communication case, in which adaptation levels decrease over the whole time horizon until they reach a level below $50 \%$. In the presence of communication policies (blue, brown, green and purple lines), adaptation levels begin to increase after some steps and reach average levels of between $65 \%$ and $80 \%$. As depicted in Figure 12, the trajectory associated with the top down policy on risk and coping ("td-rc" policy) crosses the trajectory associated with the top down policy on risk ("td-r" policy) and then crosses the trajectory associated with the people centred policy on risk ("pc-r" policy). From this point on, we observe that, on average, adaptation levels after "pc-rc" policies (people centred policies on risk and coping) are higher than adaptation levels after "pc-r" policies (people centred policies on risk) and adaptation levels after "td-rc" policies (top down policies on risk and coping) are greater than adaptation levels after "td-r" policies (top down policies on risk). Hence, risk and coping ("rc") policies outperform risk ("r") policies. To illustrate the different outcomes in more detail and to test whether mean adaptation rates differ significantly from each other, we chose to focus on two particular time steps (years) which we will use as examples in the following sections. We choose year 15 as it is long enough from the initialisation to see a differentiated impact of the policies, and year 25 as it can be considered as a long term horizon in our model. Numerical results for time steps 15 and 25 are presented in Table 5. Mann Whitney Wilcoxon rank sum tests confirmed that all mean trajectories differ significantly from one another except the "td-r" and "pc-r" policies in both time-steps and the "td-rc" and "pc-r" trajectories in time step 15, where they cross. This confirms the rankings above.

In the following, we analyze the performance of different communication policies, taking into consideration the fact that other influential variables have to be taken into account: we first examine the impact of the PMT dataset, then the impact of the network parameter and finally the impact of the most important parameters $b$ and $N$. 


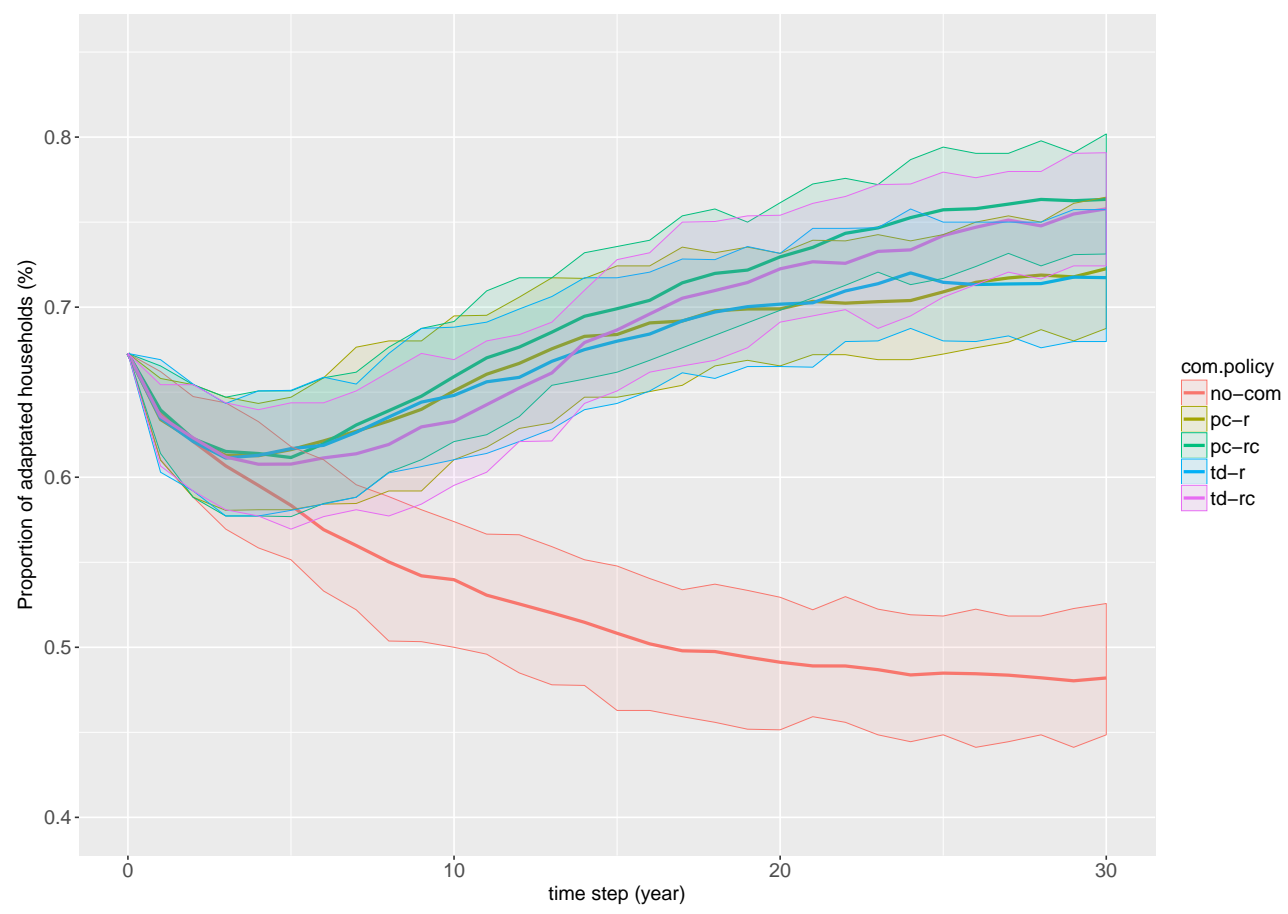

Figure 12: Proportion of adapted households under communication policies in the baseline case, evolution over a time horizon of 30 years.

\begin{tabular}{l|rr|rr}
\hline & \multicolumn{3}{c}{ Time step 15 } & \multicolumn{2}{c}{ Time step 25 } \\
Policy scenario & mean & (sd) & mean & (sd) \\
\hline no communication (nocom) & 0.51 & $(0.03)$ & 0.48 & $(0.03)$ \\
top down on risk (tdr) & 0.68 & $(0.03)$ & 0.71 & $(0.03)$ \\
top down on risk and coping (tdrc) & 0.69 & $(0.03)$ & 0.74 & $(0.03)$ \\
people centred on risk (pcr) & 0.68 & $(0.03)$ & 0.71 & $(0.03)$ \\
people centred on risk and coping (pcrc) & 0.70 & $(0.03)$ & 0.76 & $(0.03)$ \\
\hline
\end{tabular}

Table 5: Mean adaptation levels and standard deviations (in brackets) in the baseline case at time steps 15 and 25 .

\subsubsection{The importance of the psychological datasets}

Using data from the literature To assess the relative importance of the dataset explaining households' perceptions and motivations, we ran the model using a dataset from the literature. Bubeck et al. (2013) conducted a survey of 752 flood prone households along the River Rhine in Germany. Based on the PMT framework, they explained households' decision to take precautionary measures. This data was already used to assess the adaptation behaviour of Dutch households in Haer et al. (2016b). We use it in the following as a comparison to our data. In particular, we take the odds-ratios explaining the adoption of adapted building use, which are non permanent adaptation measures. We adopt the initial conditions and setups proposed by Haer et al. (2016b). Note that we change no other assumptions in the model, neither the institutional setting nor elements related to the hazard, which could be an interesting assumption in further research.

Figure 13 shows the evolution of adaptation levels over 30 years using this alternative dataset, all other parameters corresponding to the baseline case. Now, the highest mean adaptation rate is reached with the "td-rc" policy.

As an illustration, mean adaptation levels at time step 15 are listed in Table 6. Mann Whit- 


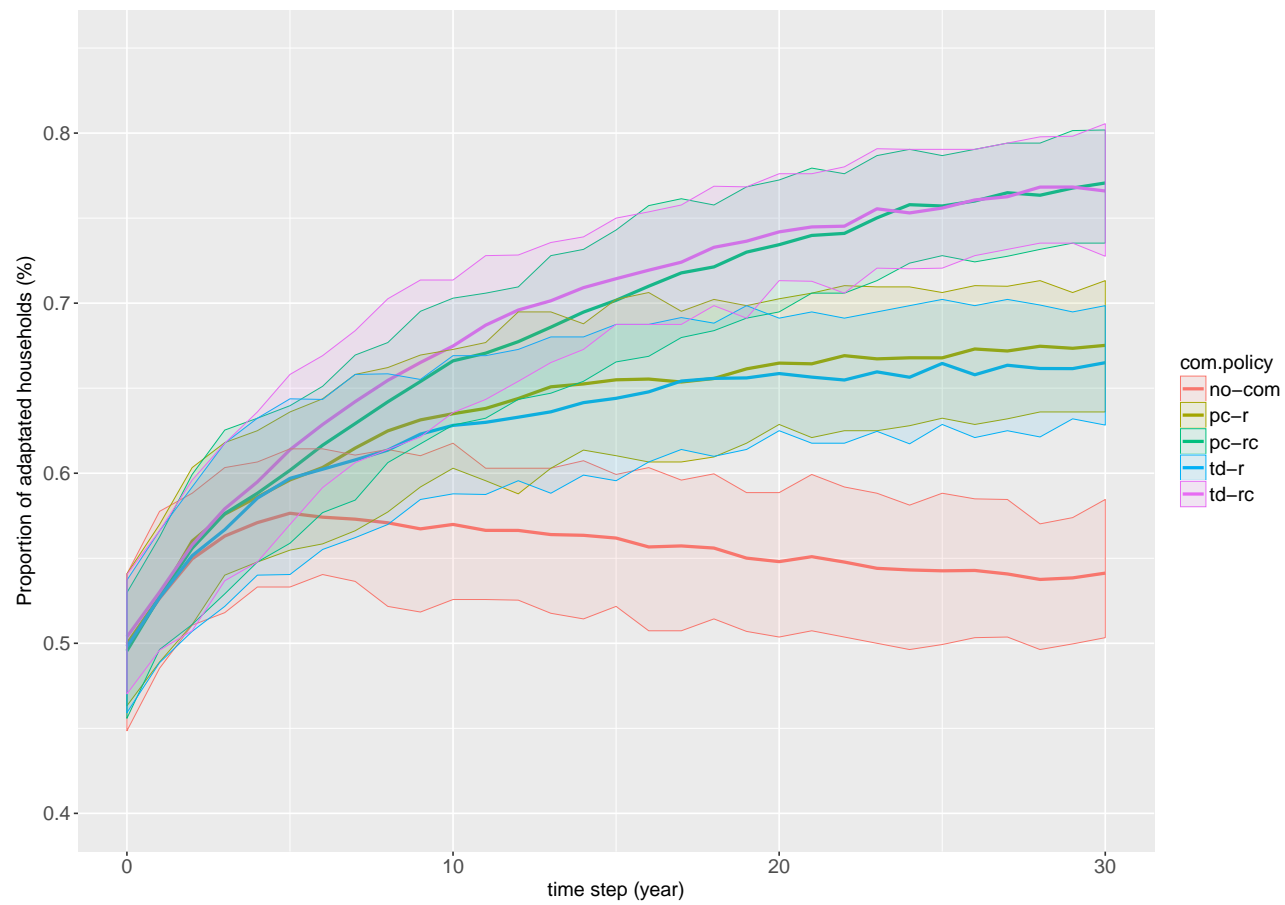

Figure 13: Proportion of adapted households under communication policies, based on Bubeck et al. 2013, evolution over a time horizon of 30 years.

\begin{tabular}{l|rr|rrr}
\hline & \multicolumn{3}{c}{ Baseline case } & \multicolumn{2}{c}{ Alternative case } \\
Policy scenario & mean & $(\mathrm{sd})$ & mean & $(\mathrm{sd})$ \\
\hline no communication (nocom) & 0.51 & $(0.03)$ & 0.56 & $(0.04)$ \\
top down on risk (tdr) & 0.68 & $(0.03)$ & 0.64 & $(0.03)$ \\
top down on risk and coping (tdrc) & 0.69 & $(0.03)$ & 0.71 & $(0.02)$ \\
people centred on risk (pcr) & 0.68 & $(0.03)$ & 0.65 & $(0.04)$ \\
people centred on risk and coping (pcrc) & 0.70 & $(0.03)$ & 0.70 & $(0.03)$ \\
\hline
\end{tabular}

Table 6: Mean adaptation levels (standard deviations in brackets) in the alternative case, with data from Bubeck et al. (2013), compared to the baseline case, at time step 15.

ney Wilcoxon rank sum tests show that all mean adaptation levels are significantly different from each other at time step 15 . At time step 25, the "td-r" policy is not signficantly different from the "pc-r" policy (as in the baseline case) but neither is the "td-rc"-"pc-rc" pair distinctive any longer.

Comparison with the baseline case Let us now compare the results from this alternative case with our results. As can be seen by comparing Figures 12 and 13, in the alternative case, adaptation rates flex to an upward trend earlier than in the baseline case. This is especially true for the "rc" policies. This could be explained with the odds ratio of the coping variables. Indeed, in Bubeck et al. (2013) the odds-ratio of the perceived efficacy of measures is greater than in our data. Combined with a distribution of lower attitude levels, this may lead to a sharper increase in adaptation rates in presence of communication policies that play on the coping variable.

Table 6 enables comparison of the simulations at time step 15. We can first compare the rankings of the different solutions: in both cases, "pc-rc" policies perform better than "pc-r" policies and "td-rc" policies perform better than "td-r" policies. However, in the 
baseline case, the "pc-rc" policy is better than the "td-rc" policy but in the alternative case, the "td-rc" policy is better than the "pc-rc" policy. Next, we can compare the levels of adaptation in both cases. It should be recalled that the initial value is not the same: it is collected data in the baseline case and a random value between zero and one in the alternative case. Although the initial value is smaller in the alternative case, it leads to higher levels of adaptation in the no communication scenario. On the other hand, "pcrc" policies result in the same absolute levels of adaptation with both datasets. These observations are true for both, time step 15 and time step 25.

To conclude this comparison, on the grounds of these two modelling cases, it is not possible to decide whether people centred risk and coping ("pc-rc") or top down risk and coping ("td-rc") policies perform best.

\subsubsection{The importance of the social network variable}

The small world network In the following, we illustrate the impact of the small world network variable at time steps 15 and 25. Figure 14 shows mean adaptation levels at
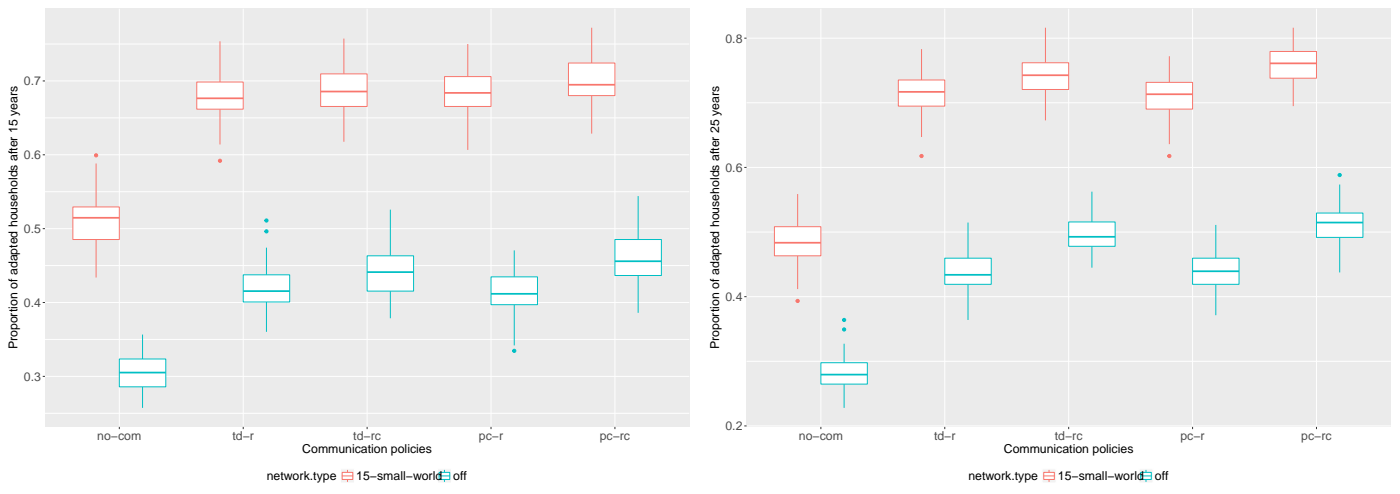

Figure 14: Boxplot of adaptation rates under communication policies at time step 15 (left panel) and at time step 25 (right panel) baseline case (red) and case with no network (blue).

time step 15 (left) and time step 25 (right). The upper (red) boxes correspond to the mean adaptation level in the baseline case, hence including a small world network, and the lower (blue) boxes to the case with no social network. The corresponding means and standard deviations at time step 15 are given in Table 7 and those at time step 25 in Table 8, compared to the baseline case. The absence of the small world network decreases

\begin{tabular}{l|rr|rrr}
\hline & \multicolumn{3}{c}{ Baseline case } & \multicolumn{2}{c}{ With no network } \\
Policy scenario & mean & $(\mathrm{sd})$ & mean & $(\mathrm{sd})$ \\
\hline no communiction (nocom) & 0.51 & $(0.03)$ & 0.30 & $(0.02)$ \\
top down on risk (tdr) & 0.68 & $(0.03)$ & 0.42 & $(0.03)$ \\
top down on risk and coping (tdrc) & 0.69 & $(0.03)$ & 0.44 & $(0.03)$ \\
people centred on risk (pcr) & 0.68 & $(0.03)$ & 0.41 & $(0.03)$ \\
people centred on risk and coping (pcrc) & 0.70 & $(0.03)$ & 0.46 & $(0.03)$ \\
\hline
\end{tabular}

Table 7: Means and standard deviations of adaptation rates at time step 15, baseline case and case with no network.

the absolute value of adaptation levels. For example, at time step 15, under the "pc-rc" policy, mean adaptation levels with the smallworld network reach $70 \%$, whereas they are only $46 \%$ on average in the absence of a social network. At time step 25, the differences 
between policies are even more pronounced: under the "pc-rc" policy, mean adaptation levels with the smallworld network reach $76 \%$, whereas they are at only $51 \%$ on average in absence of a social network. Interestingly, the ranking of the performance of the different communication policies remains the same in presence and absence of the social network.

\begin{tabular}{l|rr|rrr}
\hline \multirow{2}{*}{ Policy scenario } & \multicolumn{3}{c}{ Baseline case } & \multicolumn{2}{c}{ With no network } \\
\hline no communication (nocom) & 0.48 & $(0.03)$ & 0.28 & $(0.03)$ \\
top down on risk (tdr) & 0.71 & $(0.03)$ & 0.44 & $(0.03)$ \\
top down on risk and coping (tdrc) & 0.74 & $(0.03)$ & 0.50 & $(0.03)$ \\
people centred on risk (pcr) & 0.71 & $(0.03)$ & 0.44 & $(0.03)$ \\
people centred on risk and coping (pcrc) & 0.76 & $(0.03)$ & 0.51 & $(0.03)$ \\
\hline
\end{tabular}

Table 8: Means and standard deviations of adaptation rates at time step 25, baseline case and case with no network.

Other network types We also tested the impact of other network types, namely the network of $n$ closest neighbours and a random network, and other degrees of connection, namely $n=5, n=10$ and $n=15$. In all policy scenarios with communication policies, the network types and degrees had no impact on the simulation results. This is mainly due to the fact that the communication policies affect households randomly in the population, independently of their positions in the network. In the scenario with no communication (no-com), the effect of the type of network is very small, as shown in Figure 15, which represents adaptation rates for different network types and network degrees, at the end of the time horizon in the baseline case.

\subsubsection{Importance of the dynamic variables}

We now turn to the impact of the dynamic variables on the trajectories of individual adaptation and the efficiency of communication policies. We first discuss the impact of $b$, the adaptation duration, and then the impact of $N$, the delay before implementation of the measures.

Adaptation duration Let us consider the cases where adaptations last either 3 or 11 years on average, instead of 7 , all other variables being the same as in the baseline case. As shown in the aggregate model, decreasing $b$ will lead to lower adaptation trajectories and increasing $b$ to higher trajectories.

When $b=3$ adaptation trajectories without policies decrease to nearly $20 \%$ in year 30 , compared to slightly below $50 \%$ in the baseline case, whereas the best performing policy with $b=3$ leads to adaptation levels of only $45 \%$. Table 9 illustrates mean adaptation levels at time step 15. The ranking of the performance of policies is the same as in the baseline case and Mann Whitney Wilcoxon rank sum tests show that only the "td-r"-"pc$\mathrm{r}^{\prime \prime}$ pair is not significantly different. However, absolute adaptation levels in presence of the best performing policy are even lower than in the no communication scenario of the baseline case.

Figure 16 shows adaptation trajectories for the case where the adaptation duration is 11 years on average. When $b=11$, adaptation trajectories with no communication policy decrease to a level of above $60 \%$, compared to below $50 \%$ in the baseline case. Likewise, the adaptation level after policy intervention reaches $85 \%$ in case of the most efficient policy, compared to $75 \%$ in the baseline case. The "pc-rc" policy still performs best over the whole time horizon. At time step 15, only the "pc-rc" policy is really distinct from 


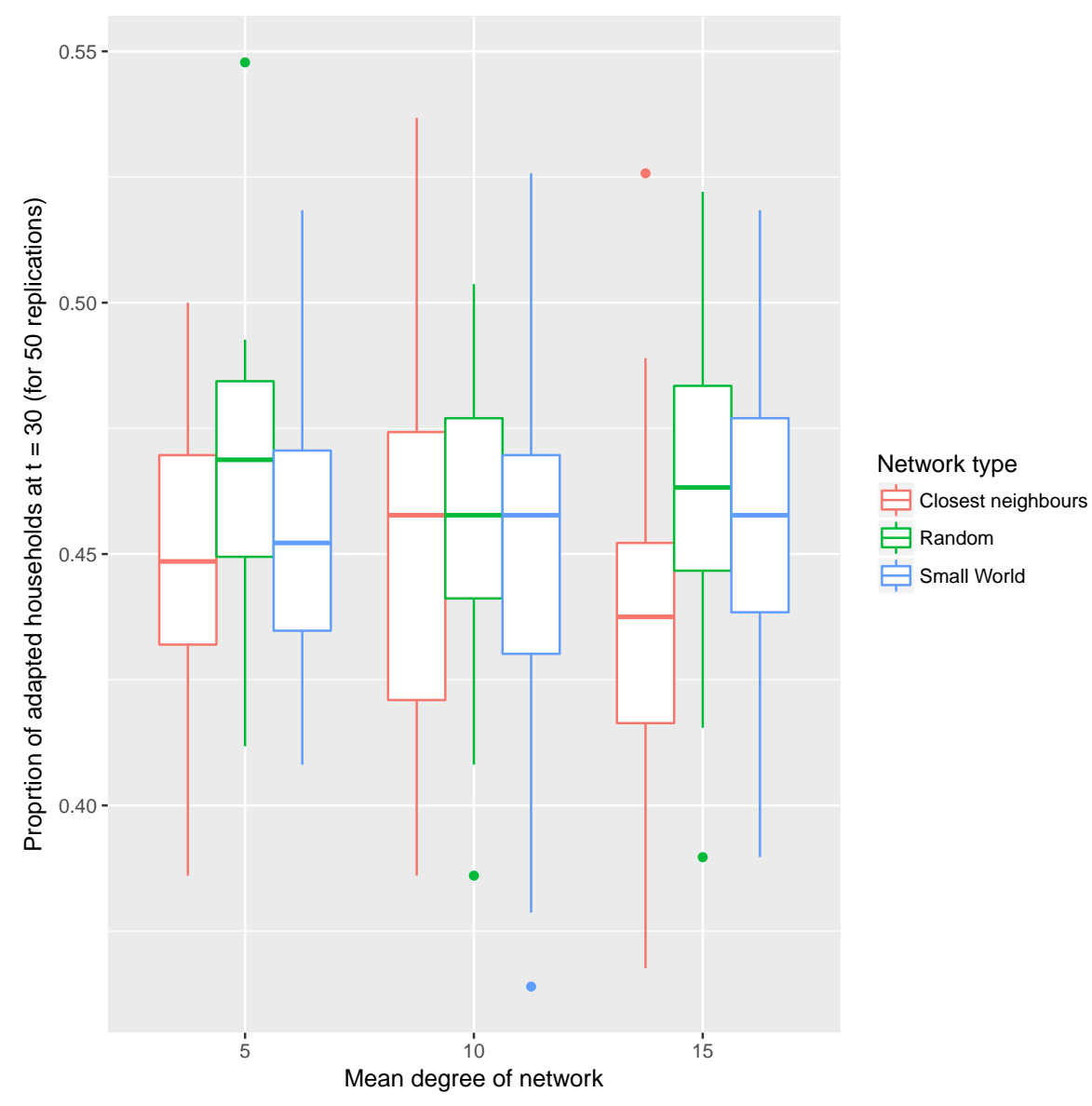

Figure 15: Boxplot of adaptation rates at the end of the time horizon in the baseline case. Comparison of the effect of different network types (in colour) and different network degrees, on the $\mathrm{x}$-axis.

the others. However, at time step 25, the results are similar to the baseline case : only the "tdr-pcr" pair is not significantly different from the others. As an illustration, the mean adaptation rates for time step 25 are given in Table 10 . The relative performance of policies is the same as before, only the absolute adaptation levels change.

Delay of implementation of the measure Let us finally discuss the case where the adaptation measure is only operational three years on average after the decision to implement it $(N=3)$. Individuals are still assumed to abandon measures on average after seven years $(b=7)$. The adaptation trajectories are shown in Figure 17 .

Note that mean adaptation levels are much lower than in the baseline case. With no communication policies, the proportion of adapted households reaches slightly more than $20 \%$ only, compared to levels approaching $50 \%$ in the baseline case. The most efficient communication policy only leads to adaptation levels of below $45 \%$ at time step 30, compared to more than $75 \%$ in the baseline case. However, as before, the overall ranking of policies does not change compared to the baseline case. Mann Whitney Wilcoxon rank sum tests show that all adaptation levels at time steps 15 and 25 are significantly different from one another, except for the "td-r" and "pc-r" pair (as in the baseline case). Table 11 illustrates mean adaptation rates compared to the baseline case at time step 15.

Comparing the results in Table 11 and in Table 7 illustrates that changing the delay of adaptation by two years has a stronger impact than switching the network off. Moreover, 


\begin{tabular}{l|rr|rrr}
\hline & \multicolumn{3}{c}{ Baseline case } & \multicolumn{2}{c}{ Duration $b=3$} \\
Policy scenario & mean & $(\mathrm{sd})$ & mean & $(\mathrm{sd})$ \\
\hline no communication(nocom) & 0.51 & $(0.03)$ & 0.24 & $(0.02)$ \\
top down on risk (tdr) & 0.68 & $(0.03)$ & 0.38 & $(0.03)$ \\
top down on risk and coping (tdrc) & 0.69 & $(0.03)$ & 0.41 & $(0.03)$ \\
people centred on risk (pcr) & 0.68 & $(0.03)$ & 0.38 & $(0.03)$ \\
people centred on risk and coping (pcrc) & 0.70 & $(0.03)$ & 0.42 & $(0.04)$ \\
\hline
\end{tabular}

Table 9: Means and standard deviations of adaptation rates at time step 15, baseline case and case where $b=3$.

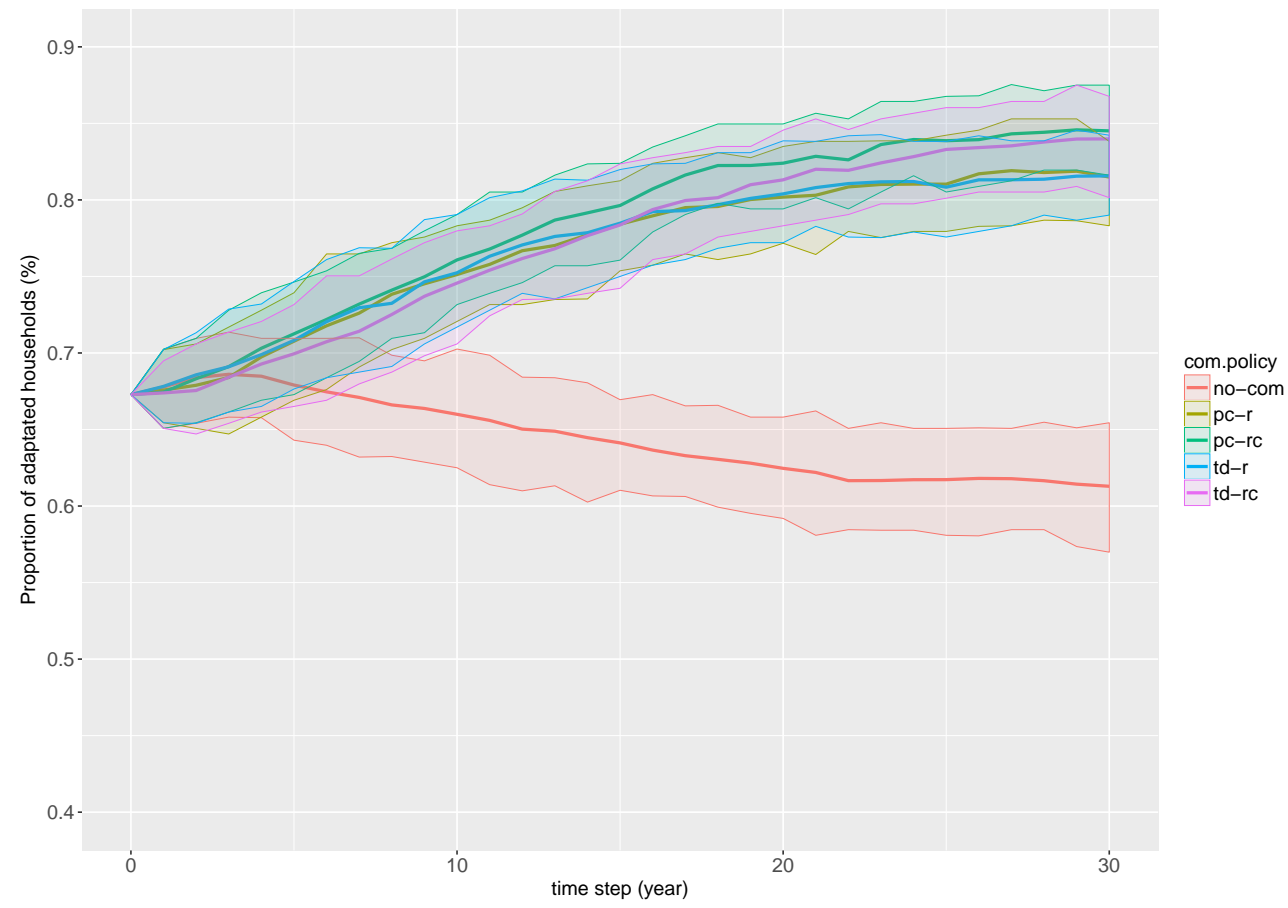

Figure 16: Proportion of adapted households under communication policies, adaptation duration, $b=11$ years.

comparing the results in Table 11 and in Table 9 shows that, in absence of communication policies, increasing the delay of adaptation by two years has a smaller impact on adaptation levels than decreasing the adaptation duration by 4 years. On the other hand, in presence of communication policies, increasing the delay of adaptation by two years has a bigger impact on adaptation levels than decreasing adaptation duration by 4 years. Finally note that the impact of increasing $N$ is similar for the alternative dataset.

\section{Conclusion}

We have built a dynamic agent based model of the adoption of individual adaptation measures against floods. Parameterized with data from an original survey conducted in the South of France, the model can be used to discuss the efficiency of different communication policies and to evaluate the importance of poorly known variables of adaptation dynamics. We can conclude that the communication policies studied here, which were proposed in the literature (Haer et al., 2016b), have quite a big impact on adaptation levels in the population. In the baseline case, communication policies increase adaptation levels by 20 


\begin{tabular}{l|rr|rrr}
\hline & \multicolumn{3}{c}{ Baseline case } & \multicolumn{2}{c}{ Duration $b=11$} \\
Policy scenario & mean & $(\mathrm{sd})$ & mean & $(\mathrm{sd})$ \\
\hline no communication (nocom) & 0.48 & $(0.0)$ & 0.62 & $(0.03)$ \\
top down on risk (tdr) & 0.71 & $(0.03)$ & 0.81 & $(0.03)$ \\
top down on risk and coping (tdrc) & 0.74 & $(0.03)$ & 0.83 & $(0.03)$ \\
people centred on risk (pcr) & 0.71 & $(0.03)$ & 0.81 & $(0.03)$ \\
people centred on risk and coping (pcrc) & 0.76 & $(0.03)$ & 0.84 & $(0.03)$ \\
\hline
\end{tabular}

Table 10: Means and standard deviations of adaptation levels when $b=11$, compared to the baseline case, at time step 25 .

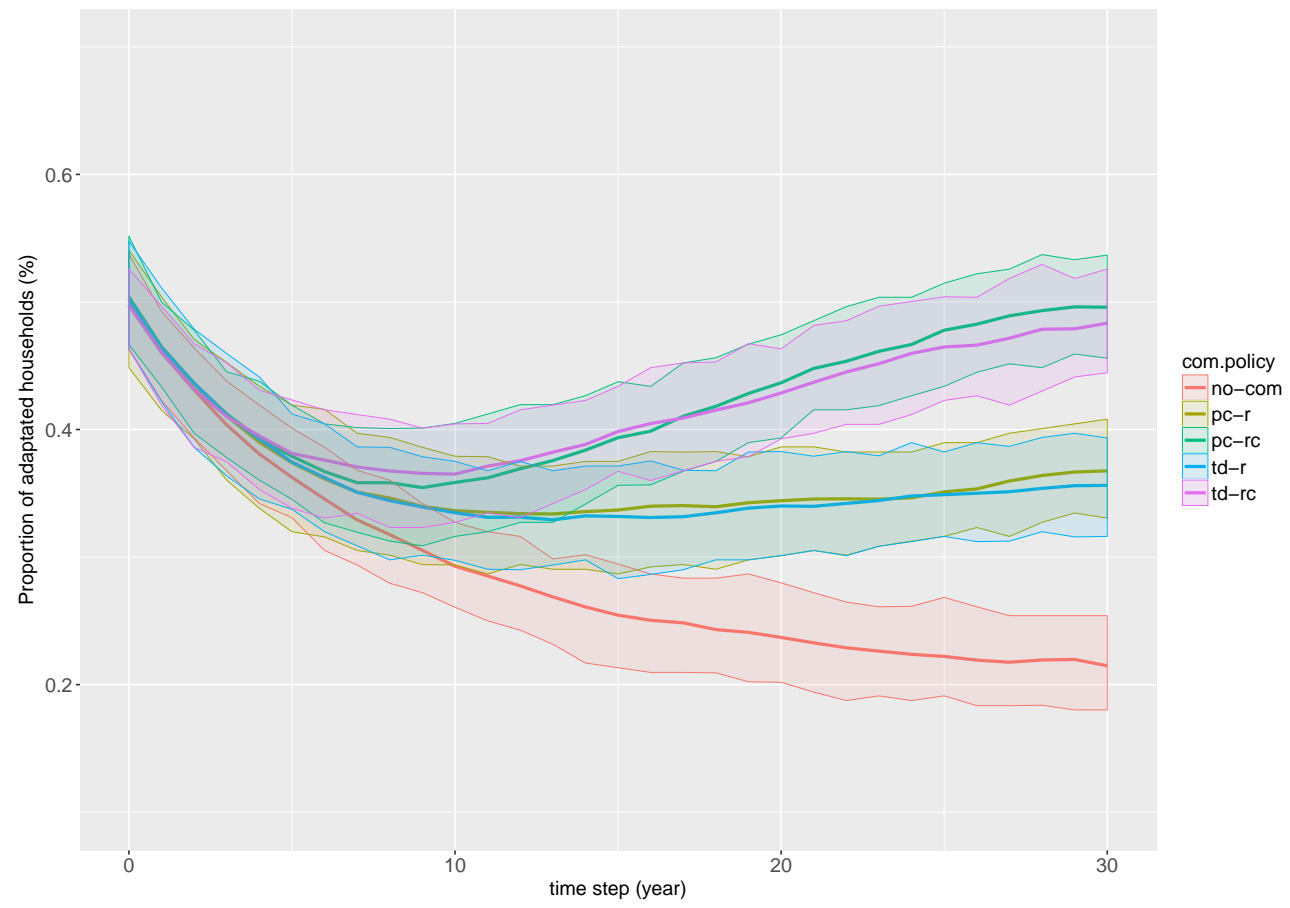

Figure 17: Proportion of adapted households under communication policies, delay of action, $N=3$ years.

percentage points at time step 15 and up to over 30 percentage points at the end of the time horizon. Because the decision model of the agents is based on the psychological protection motivation theory that decomposes the individual adaptation motivation into variables relating to threat appraisal and coping appraisal, we can draw some general lessons about different types of communication policies. In all settings, even in the short term, policies which contain information on both the risk of flooding and how to cope with it, perform better than policies which only deal with risk. Moreover people centred policies on risk and coping perform best in all scenarios based on our dataset. Although absolute levels of adapation do not change much when using an external dataset, relying on external data from other studies leads to the only case where the top down policy on risk and coping performs best.

However, conclusions about the absolute levels of adaptation, and hence on overall damage resulting from floods, should be drawn with caution. The sensitivity analysis makes it possible to target those parameters for which more information should be collected: the delay of implementation of the measure is the most important parameter in our model, before the adaptation duration. This calls for more dedicated studies on the real barriers 


\begin{tabular}{lrrrr}
\hline & \multicolumn{3}{c}{ Baseline case } & \multicolumn{2}{c}{ Delay } & $N=3$ \\
Policy scenario & mean & $(\mathrm{sd})$ & mean & $(\mathrm{sd})$ \\
\hline no communication (nocom) & 0.51 & $(0.03)$ & 0.27 & $(0.03)$ \\
top down on risk (tdr) & 0.68 & $(0.03)$ & 0.37 & $(0.03)$ \\
top down on risk and coping (tdrc) & 0.69 & $(0.03)$ & 0.39 & $(0.03)$ \\
people centred on risk (pcr) & 0.68 & $(0.03)$ & 0.37 & $(0.03)$ \\
people centred on risk and coping (pcrc) & 0.70 & $(0.03)$ & 0.40 & $(0.03)$ \\
\hline
\end{tabular}

Table 11: Means and standard deviations of adaptation rates at time step 15, baseline case compared with the dynamic case with delay of action, $N=3$ years, and adaptation duration, $b=7$ years.

to implementation and the sustained use of adaptation measures by households.

Several extensions to this article are possible: first, households could be mobile, as assumed in Haer et al. (2016b), which could lead to the abandonment of certain individual adaptation measures, namely behavioural measures. Second, adaptation behaviour could depend on the occurrence of flood events, as several studies have shown that experience is an important factor for individual adaptation. Next, we could consider different institutional settings, which would favour or hinder individual adaptation. Moreover, there could be economic incentives for individual adaptation measures, such as subsidies for the implementation of the measures. One could also consider that the policy maker has some specific targets which he/she would like to achieve in a cost efficient way: e.g. reaching a certain level of adapted households or reducing overall damage by a specified amount. $\mathrm{He} /$ she could then decide which type of policy is the best to reach this objective. Finally, it could be challenging to use the model for simulations in two different countries, with different hazard, different institutional settings and different people. ${ }^{5}$

\section{Acknowledgements:}

The authors gratefully acknowledge financial support from Irstea via the "AAP Risques" project RAZ 13.

\section{References}

Adger, W. N., Arnell, N. W., Tompkins, E. L., 2005. Adapting to climate change: perspectives across scales. Global Environmental Change 15 (2), 75 - 76.

Aven, T., Renn, O., 2010. Risk Management and Governance - Concepts, Guidelines and Applications. Springer Berlin Heidelberg.

Blanco, E., Dutcher, E. G., Haller, T., 2017. To mitigate or to adapt? collective action under asymmetries in vulnerability to losses. WP from the Faculty of Economics and Statistics, University of Innsbruck 2014-27, 1-35.

Bonté, B., Mathias, J.-D., Duboz, R., 2012. Moment approximation of infection dynamics in a population of moving hosts. PLoS ONE 7 (12), e51760.

Botzen, W. J. W., Aerts, J. C. J. H., van den Bergh, J. C. J. M., 2009. Willingness of homeowners to mitigate climate risk through insurance. Ecological Economics 68 (8), $2265-2277$.

\footnotetext{
${ }^{5}$ We thank an anonymous referee for this suggestion.
} 
Bubeck, P., Botzen, W., Kreibich, H., Aerts, J., 2013. Detailed insights into the influence of flood-coping appraisals on mitigation behaviour. Global Environmental Change 23 (5), $1327-1338$.

Bubeck, P., Botzen, W., Suu, L., Aerts, J., 2012a. Do flood risk perceptions provide useful insights for flood risk management? findings from central vietnam. Journal of Flood Risk Management 5 (4), 295-302.

Bubeck, P., Botzen, W. J. W., Aerts, J. C. J. H., 2012b. A review of risk perceptions and other factors that influence flood mitigation behavior. Risk Analysis 32 (9), 1481-1495.

Dawson, R. J., Peppe, R., Wang, M., Oct 2011. An agent-based model for risk-based flood incident management. Natural Hazards 59 (1), 167-189.

Dubbelboer, J., Nikolic, I., Jenkins, K., Hall, J., 2017. An agent-based model of flood risk and insurance. Journal of Artificial Societies and Social Simulation 20 (1), 6.

EEA, 2012. Climate change, impacts and vulnerability in europe 2012 - an indicatorbased report. Report No12/2012, European Environment Agency, Copenhagen.

Epstein, L. G., Axtell, R., 1996. Growing Artificial Societies. Brookings Institution Press, The MIT Press.

Erdlenbruch, K., Thoyer, S., Grelot, F., Kast, R., Enjolras, G., 2009. Risk-sharing policies in the context of the french flood prevention action programmes. Journal of Environmental Management 91 (2), 363 - 369.

Filatova, T., 2014. Market-based instruments for flood risk management: A review of theory, practice and perspectives for climate adaptation policy. Environmental Science \& Policy 37, 227-242.

Filatova, T., 2015. Empirical agent-based land market: Integrating adaptive economic behavior in urban land-use models. Computers, Environment and Urban Systems 54, $397-413$.

Filatova, T., Parker, D. C., van der Veen, A., 2011. The implications of skewed risk perception for a dutch coastal land market: Insights from an agent-based computational economics model. Agricultural and Resource Economics Review 40 (3), 405-423.

Glenk, K., Fischer, A., 2010. Insurance, prevention or just wait and see? public preferences for water management strategies in the context of climate change. Ecological Economics 69 (11), 2279-2291.

Grothmann, T., Reusswig, F., 2006. People at risk of flooding: why some residents take precautionary action while others do not. Natural Hazards 38, 101-120.

Haer, T., Botzen, W. J. W., de Moel, H., Aerts, J. C. J. H., 2016a. Integrating household risk mitigation behavior in flood risk analysis: An agent-based model approach. Risk Analysis, 1-16.

Haer, T., Botzen, W. W., Aerts, J. C., 2016b. The effectiveness of flood risk communication strategies and the influence of social networks-insights from an agent-based model. Environmental Science \& Policy 60, 44-52.

IRGC, 2005. Risk governance: Towards an integrative approach. white paper no 1. Tech. rep., O. Renn with an Annex by P. Graham. Geneva. International Risk Governance Council (IRGC). 
Kreibich, H., Thieken, A. H., Petrow, T., Müller, M., Merz, B., 2005. Flood loss reduction of private households due to building precautionary measures-lessons learned from the elbe flood in august 2002. Natural Hazards and Earth System Science 5 (1), 117-126.

MEDDE, 2012a. Mieux savoir pour mieux agir : principaux enseignements de la première évaluation des risques d'inondation sur le territoire français 2011. Tech. rep., Ministère de l'écologie, du développement durable, et de l'énergie,, Paris.

MEDDE, 2012b. Première évaluation nationale des risques d'inondation - principaux résultats - epri 2011. Tech. rep., Ministère de l'écologie, du développement durable, et de l'énergie, Paris.

Milne, S., Sheeran, P., Orbell, S., 2000. Prediction and intervention in health-related behavior: A meta-analytic review of protection motivation theory. Journal of Applied Social Psychology 30 (1), 106-143.

Osberghaus, D., 2017. The effect of flood experience on household mitigation-evidence from longitudinal and insurance data. Global Environmental Change 43, 126 - 136.

Poussin, J. K., Botzen, W. W., Aerts, J. C., 2014. Factors of influence on flood damage mitigation behaviour by households. Environmental Science \& Policy 40 (0), 69 - 77.

Poussin, J. K., Botzen, W. W., Aerts, J. C., 2015. Effectiveness of flood damage mitigation measures: Empirical evidence from french flood disasters. Global Environmental Change $31,74-84$.

Reynaud, A., Aubert, C., Nguyen, M., Jul 2013. Flood protective behaviours in vietnam. Geneva Pap R I-Iss P 38 (3), 547-579.

Richert, C., Erdlenbruch, K., Figuières, C., 2017. The determinants of households' flood mitigation decisions in france - on the possibility of feedback effects from past investments. Ecological Economics 131, $342-352$.

Rogers, R. W., 1975. A protection motivation theory of fear appeals and attitude change. The Journal of Psychology 91 (1), 93-114.

van Duinen, R., Filatova, T., Geurts, P., van der Veen, A., 2014. Coping with drought risk: empirical analysis of farmers' drought adaption in the south-west netherlands. Reg Environ Change.

van Duinen, R., Filatova, T., Geurts, P., Veen, A. v. d., 2015. Empirical analysis of farmers' drought risk perception: Objective factors, personal circumstances, and social influence. Risk Analysis 35 (4), 741-755.

van Duinen, R., Filatova, T., Jager, W., van der Veen, A., Nov 2016. Going beyond perfect rationality: drought risk, economic choices and the influence of social networks. The Annals of Regional Science 57 (2), 335-369.

Watts, D., Strogatz, S., 1998. Collective dynamics of 'small-world' networks. Nature 393 (6684), 440-442.

Wilensky, U., 1999. Netlogo. Center for Connected Learning and Computer-Based Modeling, Northwestern University, Evanston, IL.

URL http://ccl.northwestern.edu/netlogo/ 